\title{
Behavioural nudges increase COVID-19 vaccinations
}

https://doi.org/10.1038/s41586-021-03843-2

Received: 1 April 2021

Accepted: 21 July 2021

Published online: 2 August 2021

\section{Open access}

Check for updates

\author{
Hengchen Dai ${ }^{1,8}$, Silvia Saccardo ${ }^{2,8}$, Maria A. Han ${ }^{3}$, Lily Roh ${ }^{4}$, Naveen Raja ${ }^{4}$, Sitaram Vangala ${ }^{5}$, \\ Hardikkumar Modi ${ }^{6}$, Shital Pandya $^{6}$, Michael Sloyan $^{7}$ \& Daniel M. Croymans ${ }^{3 凶}$
}

Enhancing vaccine uptake is a critical public health challenge ${ }^{1}$. Overcoming vaccine hesitancy ${ }^{2,3}$ and failure to follow through on vaccination intentions ${ }^{3}$ requires effective communication strategies ${ }^{3,4}$. Here we present two sequential randomized controlled trials to test the effect of behavioural interventions on the uptake of COVID-19 vaccines. We designed text-based reminders that make vaccination salient and easy, and delivered them to participants drawn from a healthcare system one day (first randomized controlled trial) ( $n=93,354$ participants; clinicaltrials number NCT04800965) and eight days (second randomized controlled trial) $(n=67,092$ individuals; clinicaltrials number NCT04801524) after they received a notification of vaccine eligibility. The first reminder boosted appointment and vaccination rates within the healthcare system by $6.07(84 \%)$ and $3.57(26 \%)$ percentage points, respectively; the second reminder increased those outcomes by 1.65 and 1.06 percentage points, respectively. The first reminder had a greater effect when it was designed to make participants feel ownership of the vaccine dose. However, we found no evidence that combining the first reminder with a video-based information intervention designed to address vaccine hesitancy heightened its effect. We performed online studies ( $n=3,181$ participants) to examine vaccination intentions, which revealed patterns that diverged from those of the first randomized controlled trial; this underscores the importance of pilot-testing interventions in the field. Our findings inform the design of behavioural nudges for promoting health decisions ${ }^{5}$, and highlight the value of making vaccination easy and inducing feelings of ownership over vaccines.
Vaccines have been crucial for eradicating or controlling several deadly infectious diseases ${ }^{1}$. However, mobilizing people to get vaccines remains a challenge. Low or delayed vaccination uptake continues to threaten global health, and can lead to outbreaks of vaccine-preventable diseases ${ }^{6}$. Developing evidence-based communication strategies to enhance voluntary vaccine uptake is therefore $\mathrm{critical}^{4}$. Previous work suggests two major approaches to increasing vaccinations ${ }^{3}$. The first aims to boost vaccine uptake intentions among those who are uncertain about vaccination. Given that changing intentions is insufficient ${ }^{7}$, the second approach involves helping people to follow through on their vaccination intentions and overcome sources of friction, such as forgetfulness ${ }^{8}$, hassle $\operatorname{costs}^{9}$ and procrastination ${ }^{10,11}$.

These approaches could help to increase vaccination rates in the context of the current COVID-19 pandemic ${ }^{12}$, which has had unprecedented $\operatorname{costs}^{13}$. Despite the growing availability of COVID-19 vaccines, $30 \%$ of US adults were still either unwilling or uncertain about getting the COVID-19 vaccine in late June 2021, and the hesitancy rate was similarly high in several other countries that had vaccines available ${ }^{14}$. Barriers to action may further lower vaccination rates among those who intend to get inoculated.

Nudges, defined as interventions that alter 'people's behavior in a predictable way without forbidding any options or significantly changing economic incentives ${ }^{15}$, could improve the uptake of COVID-19 vaccines $^{16}$. Low-cost behavioural interventions such as these have been effectively applied to other health-related decisions ${ }^{5}$, such as healthy eating ${ }^{17}$, exercising ${ }^{18}$ and influenza vaccinations ${ }^{19-21}$. To maximize vaccine uptake, it is critical to understand how to best design behavioural interventions to boost intentions to get vaccinated, remove barriers to following through on good intentions or both ${ }^{3}$.

Here we report data from two sequential large-scale randomized controlled trials (RCTs) that investigate whether nudging people to get vaccinated, using reminders that are carefully designed to reduce barriers to following through, can improve the uptake of COVID-19 vaccines. Reminders are a popular nudge ${ }^{22}$ and have proven effective across policy-relevant domains ${ }^{8,20,23,24}$. We further examine the benefits of combining our reminders with additional interventions, including

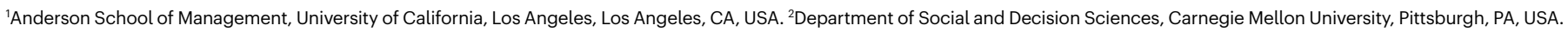
${ }^{3}$ Department of Medicine, David Geffen School of Medicine, University of California, Los Angeles, Los Angeles, CA, USA. ${ }^{4}$ Office of Population Health and Accountable Care, University of

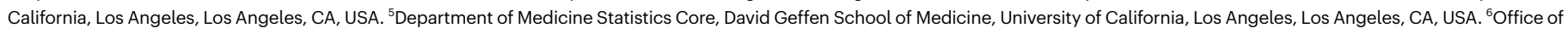

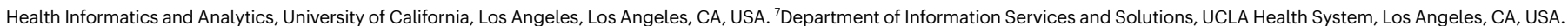

${ }^{8}$ These authors contributed equally: Hengchen Dai, Silvia Saccardo. ${ }^{\bowtie}$ e-mail: dcroymans@mednet.ucla.edu 


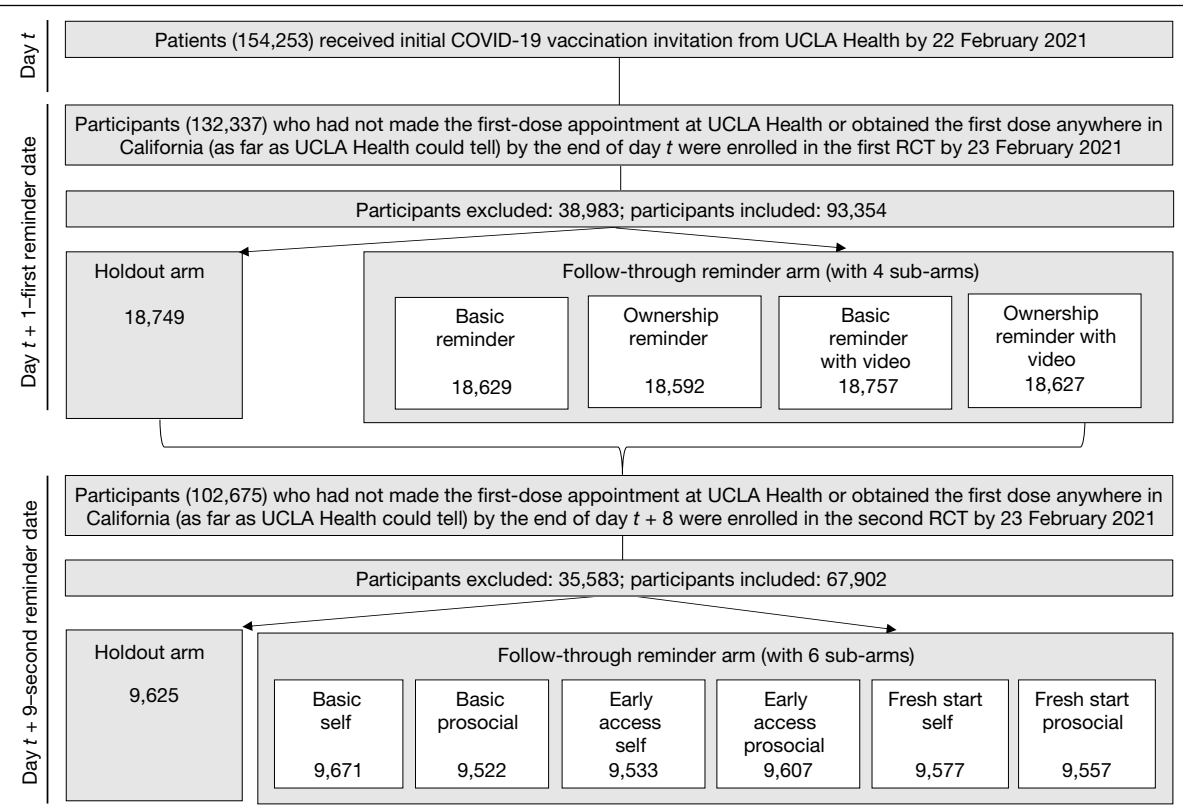

Fig. 1 Timeline, assessment for eligibility and randomization of two sequential RCTs. Timeline, eligibility for enrolment, the total number of participants excluded from the analysis, the total number of participants included in the analysis, and the number of participants who were randomized into each condition and included in the analysis are displayed here for the first and second RCTs. $t$ is the date on which participants received the initial invitation to take up a COVID-19 vaccine from UCLA Health. The first reminder date fell on the first weekday after the initial invitation was sent, and the second reminder date fell on the first weekday after the eighth day following the initial invitation. Exceptions were that participants who received the initial invitation during 19-29 January 2021 were enrolled in the first RCT on 1 February 2021 and the second RCT on 9 February 2021, owing to the delay in setting up the

(1) behaviourally informed messaging designed to amplify individuals' desire to get vaccinated and (2) a traditional information-provision intervention aimed at correcting the misconceptions that drive vaccine hesitancy ${ }^{25,26}$. Testing the effects of interventions on actual uptake of COVID-19 vaccines extends previous work that has studied hypothetical interventions ${ }^{27,28}$.

\section{Promoting vaccine uptake}

We conducted two preregistered RCTs at University of California, Los Angeles (UCLA) Health ('Data availability' in Methods). Participants in these RCTs were drawn from the UCLA Health primary and speciality care attributed patient list. Starting from 29 January 2021, once patients became eligible for the COVID-19 vaccine, UCLA Health sent them an initial invitation to schedule their vaccination appointment. On the first weekday after the initial invitation (hereafter, the first reminder date'), we enrolled eligible patients (hereafter, 'participants') in the first RCT. On the first weekday after the eighth day following the initial invitation (hereafter, the 'second reminder date'), we enrolled participants eligible for the second RCT into it. The timeline and eligibility criteria are provided in 'Enrolment and eligibility for RCTs' in Methods; Fig. 1 shows the timeline, eligibility and randomization of the two RCTs.

In both RCTs, we randomized whether participants received text-message-based reminders or not. All reminders shared two elements that were intended to address two barriers to action. First, all reminders made vaccination top of mind to curb forgetfulness and prompt people to adopt the target behaviour ${ }^{8,22}$. Second, all reminders sought to reduce inconvenience as a potential source of friction ${ }^{22}$ by including a link to the appointment-scheduling website and allowing participants to easily book their appointment immediately. infrastructure needed to run the RCTs. In the first RCT, 38,983 participants were sequentially excluded from the analysis, including (1) 33,533 individuals who obtained the first dose before the first reminder date according to the vaccination records UCLA Health could access on 25 May 2021; (2) 5,392 individuals who made the first-dose appointment at UCLA Health before 15:00 $\mathrm{h}$ on the first reminder date; and (3) 58 individuals who were under 18 years old. In the second RCT, 35,583 participants were sequentially excluded from the analysis, including (1) 35,127 individuals who obtained the first dose before the second reminder date according to the vaccination records UCLA Health could access on 25 May 2021; (2) 408 individuals who made the first-dose appointment at UCLA Health before 15:00 $\mathrm{h}$ on the second reminder date; and (3) 48 individuals who were under 18 years old.

Our primary outcome was whether participants scheduled their first-dose appointment at UCLA Health within six days of receiving a text reminder. Our secondary outcome was whether participants obtained the first dose at UCLA Health within four weeks of the reminder; the reasoning behind these time windows is given in 'Outcome measures for RCTs' in Methods.

We focus our data reporting on participants who were enrolled in the RCTs by 23 February 2021, as specified in our preregistration. All exclusion criteria and analyses were preregistered ('Enrolment and eligibility for RCTs' in Methods, Supplementary Information sections 1.1 and 1.3).

\section{First-reminder RCT}

On the first reminder date, we randomly assigned participants enrolled in the first RCT at a 4:1 ratio to the 'follow-through reminder' arm, in which they received a text reminder at 15:00 $\mathrm{h}$ that encouraged them to schedule a vaccination appointment, or to the 'holdout' arm, in which they did not get a reminder.

We nested a $2 \times 2$ factorial design within the follow-through reminder arm to test whether reminders become more effective when combined with another behaviourally informed intervention to motivate action and/or with an information intervention that aims at shifting vaccination intentions.

The first factor varied whether the reminder attempted to further amplify people's desire to act by inducing feelings of psychological ownership over the vaccine ${ }^{29,30}$. Reminders containing the ownership intervention (designated 'ownership reminder' and 'ownership reminder with video') indicated the vaccine had 'just been made available for you' and encouraged participants to 'claim your dose'. We used online experiments to confirm that such language would make participants feel more strongly that the vaccine was already theirs (ordinary least 


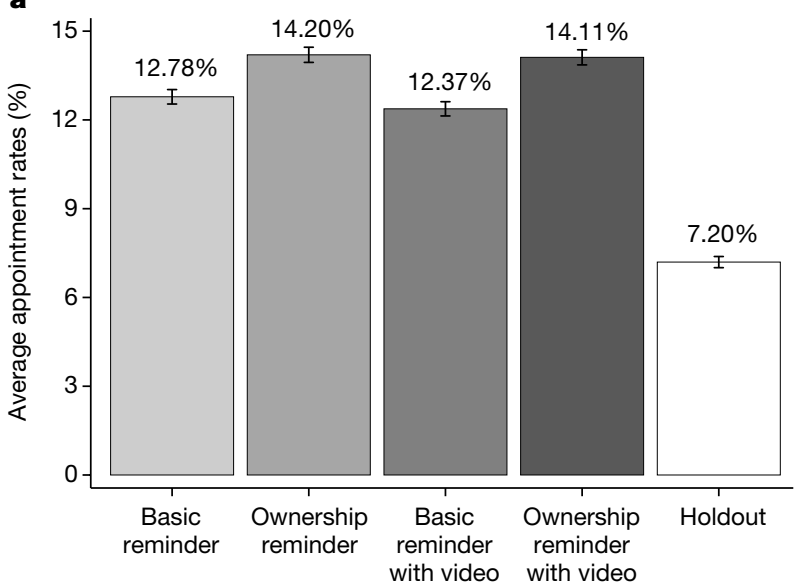

Fig. 2 | Appointment and vaccination rates at UCLA Health by condition for the first RCT. a, b, Proportion of participants in each condition who scheduled an appointment for the first dose of the COVID-19 vaccine at UCLA Health between 15:00 $\mathrm{h}$ on the first reminder date and 23:59 $\mathrm{h}$ on the fifth day following the first reminder date (a) and the proportion of participants in each b

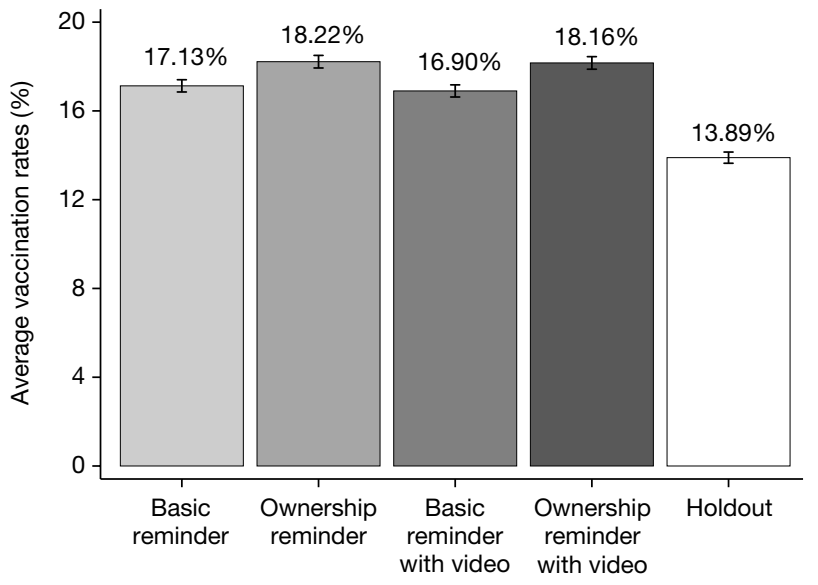

condition who obtained the first dose of the COVID-19 vaccine at UCLA Health within four weeks of the first reminder date (b). Error bars represent \pm 1 s.e.m. The number of participants in each condition (from left to right in each panel) is $18,629,18,592,18,757,18,627$ and 18,749 .

The gap between the follow-through reminder and holdout arms in vaccinations at UCLA Health persisted for eight weeks (Fig. 3), which suggests that reminders increased the number of vaccinated participants for as long as we observed (rather than only accelerating vaccinations). Notably, even if the holdout arm eventually caught up after the two months we observed, accelerating vaccination still benefits society $^{34}$.

Within the follow-through reminder arm, adding the ownership language to the reminder further increased appointment and vaccination rates at UCLA Health by 1.51 and 1.09 percentage points, respectively (Extended Data Table 2), compared to the $12.58 \%$ appointment rates and $17.01 \%$ vaccination rates among people who received a reminder without such language. By contrast, we found no evidence that inviting participants to watch the video improved either outcome variable, relative to reminders without a video (Extended Data Table 2).

The average effect of a reminder held for both participants who received the influenza shot in either of the two recent seasons $(n=46,757)$ and those who did not $(n=46,597)$ (Fig. 4$)$ but was larger among the former than the latter group, by 4.4 percentage points for appointments (OLS regression, $B=0.044$, s.e. $=0.004, P<0.001$ for the interaction) and 2.3 percentage points for vaccinations at UCLA Health (OLS regression, $B=0.023$, s.e. $=0.006, P<0.001$ for the interaction) (Supplementary Table 6).

Because our sample consists of predominantly elderly and white participants, we confirmed (Fig. 4) that the effects of follow-through reminders and ownership language largely held for racial and ethnic minorities as defined in Fig. $4(n=29,784)$ and participants under 65 years old $(n=9,279)$. Notably, the average effects of follow-through reminders on both appointments and vaccinations were comparable across white $(n=49,909)$, Hispanic $(n=10,624)$, Black $(n=5,109)$ and Asian $(n=7,553)$ participants (Extended Data Table 2$)$. Identifying solutions to improving vaccine uptake among racial and ethnic minority groups is critical, as these groups have been disproportionately hurt by the COVID-19 pandemic ${ }^{35}$ and tend to experience increased vaccine hesitancy ${ }^{36}$.

\section{Second-reminder RCT}

Participants who did not schedule their vaccine appointment a few days after the first reminder may have forgotten about it, been procrastinating or been more hesitant than those who got vaccinated. We conducted the second RCT to study the effect of sending these 


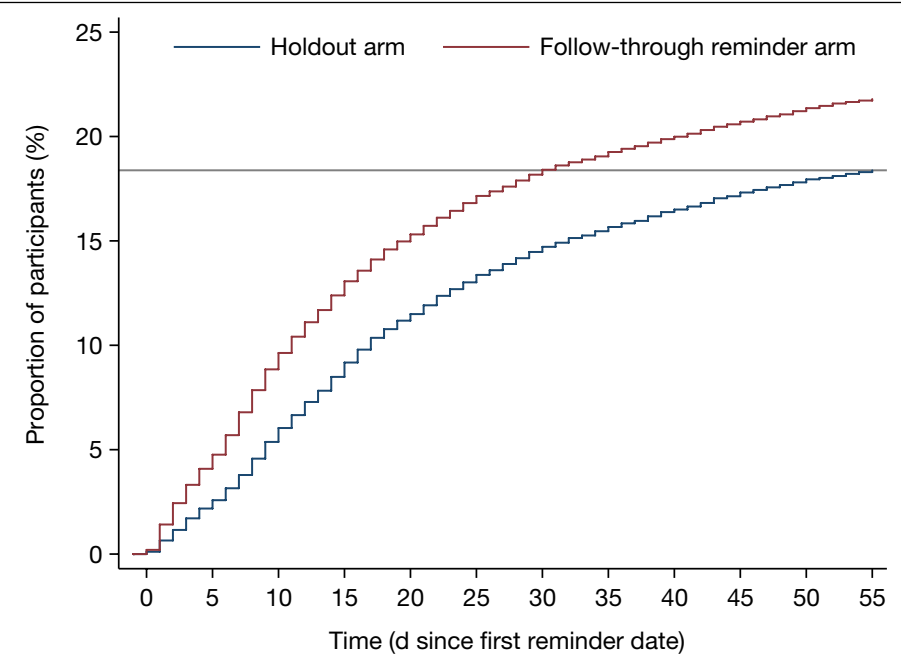

Fig. 3 | Kaplan-Meier curves reflecting the proportion of participants who had obtained the first dose at UCLA Health by a given day after the first reminder date in the first RCT. Kaplan-Meier curves tracking the percentage of participants in the holdout arm (blue) $(n=18,749)$ versus the follow-through reminder arm (red) $(n=74,605)$ of the first RCT who had obtained the first dose of COVID-19 vaccine at UCLA Health by a given day from the first reminder date ( 0 on the $x$ axis) onward. All participants were right-censored at 55 days after the first reminder date. The solid horizontal line indicates that $18.38 \%$ of participants in the holdout arm had obtained the first dose at UCLA Health by the end of 55 days after the first reminder date.

participants a second text reminder. On the second reminder date, we randomized eligible participants at a 6:1 ratio to receive another text message at 15:00 $\mathrm{h}$ that reminded them of vaccine availability and providing easy access to the scheduling website (the follow-through reminder arm) or to not receive the text message (the holdout arm).

To harness other psychological principles to motivate people to act, we randomized participants within the follow-through reminder arm to receive one of six messages that leveraged additional behavioural insights ('Design of the second-reminder RCT' in Methods). Following the preregistration, we present only the average effect of all text reminders combined relative to the holdout arm.

Our analysis includes 67,092 participants $(43.5 \%$ male, $52.6 \%$ white (excluding Hispanic or Latino), average age $=73.7$, s.d. $=10.0$ ). Study arms were well-balanced on demographic characteristics (Extended Data Table 3).

Getting a second reminder increased participants' likelihood of scheduling the first-dose appointment within six days by 1.65 percentage points (53.36\%) and obtaining the first dose at UCLA Health within four weeks by 1.06 percentage points $(17.23 \%)$, relative to the $3.10 \%$ appointment rates and $6.16 \%$ vaccination rates in the holdout arm (Extended Data Table 4). All reminder types boosted appointments and vaccinations (Extended Data Table 4). Although small, these effects are noteworthy, as they are documented within a more hesitant population (as participants in the second RCT had not scheduled an appointment after two notifications and had been eligible for COVID-19 vaccines in California for some time).

\section{Effect on vaccination anywhere}

Because the text reminders made eligibility at UCLA Health salient and reduced barriers to appointment scheduling at UCLA Health, we have focused on appointments and vaccinations at UCLA Health as our outcome measures. We also investigated the effect of receiving a text reminder on whether participants received the first dose inside or outside UCLA Health (hereafter, 'anywhere') within four weeks of getting a reminder (Supplementary Information section 1.5).
For the first RCT, we find that reminders increased vaccinations anywhere by 2.1 percentage points, relative to a baseline of $31.85 \%$ in the holdout arm (OLS regression, $B=0.021$, s.e. $=0.004, P<0.001$, $n=93,354)$ (Supplementary Table 22). In addition, adding (versus not) the ownership language increased vaccinations anywhere by an additional 0.9 percentage points (OLS regression, $B=0.009$, s.e. $=0.003$, $P=0.010$ without multiple comparison adjustment and $P=0.020$ with a Holm-Bonferroni correction ${ }^{37}, n=74,605$ ) (Supplementary Table 22). The fact that the effect of receiving one reminder on vaccinations at any location could last one month is notable, considering that participants may have been exposed to numerous sources of communication about the vaccine during this period.

Receiving a second reminder increased vaccination rates anywhere by 1.0 percentage points two weeks after the second reminder date (OLS regression, $B=0.010$, s.e. $=0.004, P=0.008, n=67,092$ ) (Supplementary Table 24), relative to a baseline of $12.04 \%$ in the holdout arm. Although this effect was not statistically significant at four weeks (OLS regression, $B=0.007$, s.e. $=0.004, P=0.127, n=67,092$ ) (Supplementary Table 23), sending a second text reminder can still contribute to accelerating vaccinations and avoiding unnecessary infections. It is also worth noting that, had we designed the reminders to remove barriers to getting vaccinated at a broad set of locations (rather than focusing on UCLA Health), our reminders might have exhibited larger effects on vaccination anywhere.

\section{Vaccination intentions versus actual uptake}

To inform policy, researchers often use surveys of intentions to evaluate the effectiveness of interventions aimed at encouraging vaccine uptake $^{3,27,28}$. Given that intentions do not always reflect real behaviours ${ }^{7}$, we tested how the interventions deployed in our first RCT affected vaccination intentions and explored whether hypothetical responses would match actual behavioural responses.

We ran three preregistered experiments on Amazon's Mechanical Turk and Prolific Academic: two concurrently to the first RCT in February 2021 and one as a replication in April 2021 (total $n=3,181$ ). We randomized participants to receive one of the four reminders from the first RCT, asking about their intentions to get vaccinated using different questions on a seven-point scale ('Procedures for online experiments' in Methods). In contrast to the patterns observed in the first RCT, the video intervention resulted in a small-but statistically significant-increase in people's self-reported interest in getting the vaccine; however, we found no evidence that adding ownership language increased vaccination intentions (Extended Data Table 5).

The discrepancy between laboratory and field data (Extended Data Table 6) is unlikely to be driven by differences in political attitudes between samples ${ }^{38}$, as the aforementioned findings about video and ownership interventions generally held both for those who self-identified as 'Democrat' and as 'Republican' online (Extended Data Table 5). One potential explanation for these discrepant findings is that, although we could require all online participants to watch the video, less than $21 \%$ of the participants in the first RCT opted to watch it (Supplementary Information section 1.3.4), possibly because of being too busy or active avoidance of information ${ }^{39}$. Another possibility is that COVID-19 vaccine intentions were harder to change outside of a controlled online experiment, where various sources of information compete for people's attention. As for the lack of evidence that ownership language affected vaccination intentions, it could be that individuals did not anticipate the motivating power of such language in hypothetical settings. Whereas the differences in sample characteristics and measurement (Extended Data Table 6) do not allow us to pinpoint the drivers of the discrepancy between our online studies and the first RCT, these results suggest that hypothetical responses to behavioural nudges should be taken with caution. 


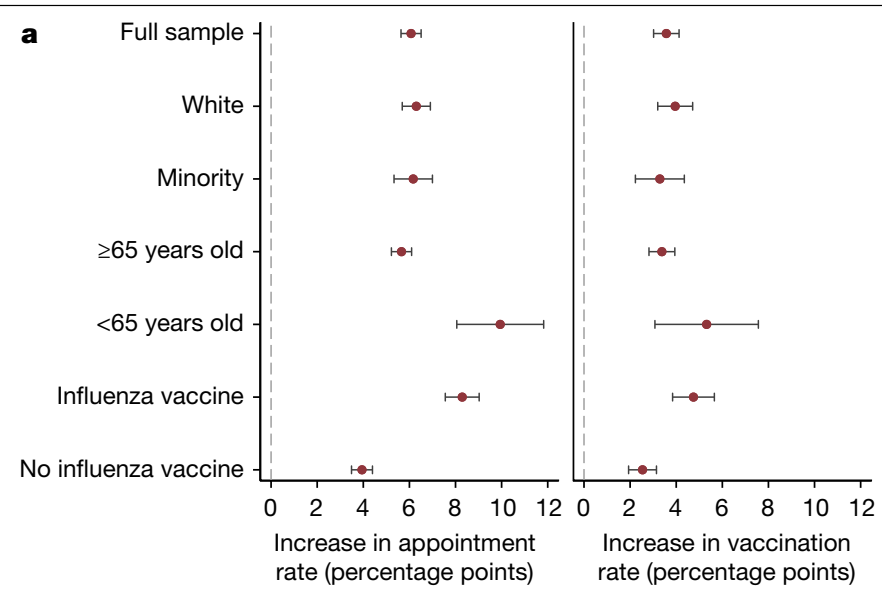

Fig. 4 | Regression-estimated increase in appointments and vaccinations induced by reminders. $\mathbf{a}, \mathbf{b}$, Regression-estimated increase in appointment rates at UCLA Health within six days of the first reminder date (left panel in a, b) and vaccination rates at UCLA Health within four weeks of the first reminder date (right panel in $\mathbf{a}, \mathbf{b}$ ), induced by receiving a reminder (versus holdout) (a) and by receiving a reminder with ownership language (versus one without) (b) across participant subgroups in the first RCT. The full sample referred to 93,354 participants included in the analysis of the first RCT. 'White', subsample including 49,909 participants who identified as white (excluding Hispanic or Latino individuals); 'minority', subsample includes 29,784 participants who identified as Asian, Black, American Indian or Alaska Native, Native Hawaiian or

\section{Discussion}

Our research highlights that behavioural science insights can increase and speed up COVID-19 vaccinations at close-to-zero marginal cost. Text-based reminders designed to overcome barriers to scheduling can effectively encourage vaccinations across different demographic groups, with effects persisting for at least eight weeks. These effects are heightened when follow-through reminders leverage psychological ownership, making people feel that a dose of the vaccine belongs to them. However, we find no evidence that combining reminders with a video-based information intervention further increases vaccination, which suggests that more work is needed to uncover when information interventions can help to overcome vaccine hesitancy. Additional analyses of our RCT sample reveal that only about $10 \%$ of participants did not keep or show up for their first-dose appointment, and approximately $90 \%$ of participants who received the first dose at UCLA Health scheduled their second dose (Supplementary Information section 1.6). Thus, the biggest barrier to increasing COVID-19 vaccinations is getting participants to schedule the first-dose appointment.

Our research has implications for enhancing the uptake of life-saving vaccines in general, as it highlights the power of making vaccination easy and eliciting feelings of ownership over the vaccine. Although promoting vaccinations at scale requires a multifaceted approach, our findings suggest that behavioural nudges could be an important strategy to consider. If sent to all 263 million adults in the USA ${ }^{40}$, and assuming the same absolute effect size observed in our first RCT would hold for the $60 \%$ of US adults who did not immediately obtain the vaccine ${ }^{41}$, our follow-through reminders could result in 3.31-5.68 million extra people getting vaccinated within a month of the reminder. This estimated range is based on the average effect of receiving the first reminder on vaccination rates anywhere (that is, $60 \% \times 263$ million $\times 2.1$ percentage points) versus at UCLA Health $(60 \% \times 263$ million $\times 3.6$ percentage points $)$. Similarly, reminders with the ownership framing would motivate 1.42-1.74 million extra people to get vaccinated than reminders without such framing (that is, $60 \% \times 263$ million $\times 0.9$ percentage points $-60 \% \times 263$ million $\times 1.1$ percentage points).

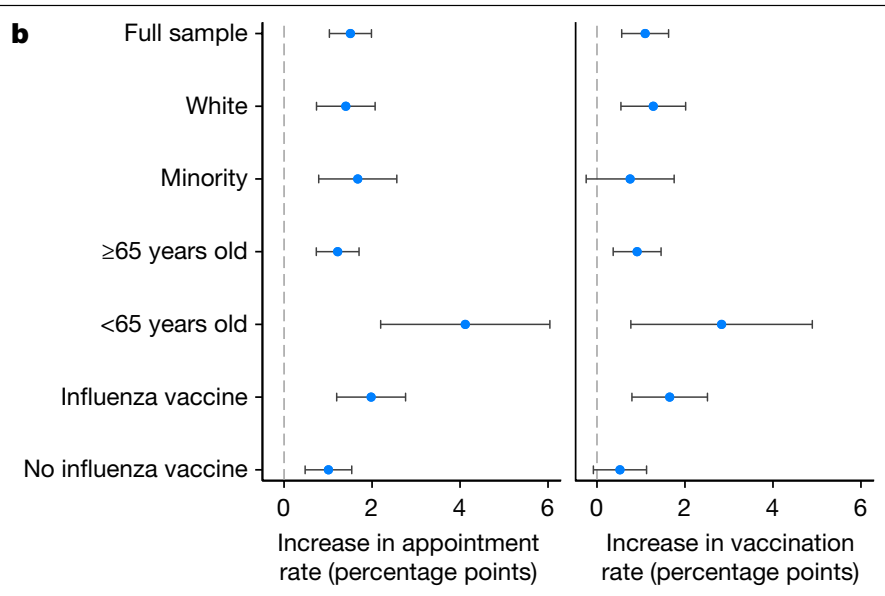

Pacific Islander, other race (excluding participants whose race was unknown), and/or Hispanic or Latino. The ‘ $\geq 65$ years old' subgroup includes 84,075 participants who were at least 65 years old; the ' $<65$ years old' subgroup includes 9,279 participants under 65 years old. The 'influenza vaccine' subgroup includes 46,757 participants who received the influenza vaccine in either of two recent influenza seasons; the 'no influenza vaccine' subgroup includes 46,597 participants who did not receive an influenza vaccine in two recent influenza seasons. Extended Data Table 2, Supplementary Tables 3, 5, 6, $10,11,13$ provide complete OLS regression results graphed here and the corresponding sample sizes. Error bars represent $95 \%$ confidence intervals of estimated increases.

The insights from this work could inform strategies to motivate health-related behaviours more broadly, such as scheduling preventive care tests or participating in health-related programs. To that end, the discrepancy observed between our RCTs and online studies highlights the value of pilot-testing interventions in the field before deploying them at scale. As policymakers, public health experts and organizations strive to develop communication strategies to promote health-related behaviours, we hope that the effective interventions documented in our research-and behavioural science more generally-can become part of their toolbox.

\section{Online content}

Any methods, additional references, Nature Research reporting summaries, source data, extended data, supplementary information, acknowledgements, peer review information; details of author contributions and competing interests; and statements of data and code availability are available at https://doi.org/10.1038/s41586-021-03843-2.

1. Greenwood, B. The contribution of vaccination to global health: past, present and future. Phil. Trans. R. Soc. Lond. B 369, 20130433 (2014).

2. MacDonald, N. E. \& the SAGE Working Group on Vaccine Hesitancy. Vaccine hesitancy: definition, scope and determinants. Vaccine 33, 4161-4164 (2015).

3. Brewer, N. T., Chapman, G. B., Rothman, A. J., Leask, J. \& Kempe, A. Increasing vaccination: putting psychological science into action. Psychol. Sci. Public Interest 18, 149-207 (2017).

4. Jarrett, C., Wilson, R., O'Leary, M., Eckersberger, E. \& Larson, H. J. Strategies for addressing vaccine hesitancy - a systematic review. Vaccine 33, 4180-4190 (2015).

5. Patel, M. S., Volpp, K. G. \& Asch, D. A. Nudge units to improve the delivery of health care. N. Engl. J. Med. 378, 214-216 (2018).

6. Jansen, V. A. A. et al. Measles outbreaks in a population with declining vaccine uptake. Science 301, 804 (2003).

7. Sheeran, P. Intention-behavior relations: a conceptual and empirical review. Eur. Rev. Soc. Psychol. 12, 1-36 (2002).

8. Karlan, D., McConnell, M., Mullainathan, S. \& Zinman, J. Getting to the top of mind: how reminders increase saving. Manage. Sci. 62, 3393-3411 (2016).

9. Bhargava, S. \& Manoli, D. Psychological frictions and the incomplete take-up of social benefits: evidence from an IRS field experiment. Am. Econ. Rev. 105, 3489-3529 (2015).

10. Shu, S. B. \& Gneezy, A. Procrastination of enjoyable experiences. J. Mark. Res. 47, 933-944 (2010).

11. Rogers, T., Milkman, K. L., John, L. K. \& Norton, M. I. Beyond good intentions: prompting people to make plans improves follow-through on important tasks. Behav. Sci. 1, 33-41 (2015). 
12. Wood, S. \& Schulman, K. Beyond politics-promoting COVID-19 vaccination in the United States. N. Engl. J. Med. 384, e23 (2021).

13. Cutler, D. M. \& Summers, L. H. The COVID-19 pandemic and the $\$ 16$ trillion virus. J. Am Med. Assoc. 324, 1495-1496 (2020).

14. Shelburne, P. U.S. lags behind global counterparts in driving down vaccine hesitancy, https://morningconsult.com/global-vaccine-tracking (accessed on 3 July 2021).

15. Thaler, R. H. \& Sunstein, C. NUDGE: Improving Decisions About Health, Wealth, and Happiness, vol. 47 (Penguin, 2009).

16. Volpp, K. G., Loewenstein, G. \& Buttenheim, A. M. Behaviorally informed strategies for a national COVID-19 vaccine promotion program. J. Am. Med. Assoc. 325, 125-126 (2021).

17. Wisdom, J., Downs, J. S. \& Loewenstein, G. Promoting healthy choices: information versus convenience. Am. Econ. J. Appl. Econ. 2, 164-178 (2010).

18. Milkman, K. L. et al. Megastudies improve the impact of applied behavioral science. Nature (in the press).

19. Chapman, G. B., Li, M., Colby, H. \& Yoon, H. Opting in vs opting out of influenza vaccination. J. Am. Med. Assoc. 304, 43-44 (2010).

20. Milkman, K. L. et al. A megastudy of text-based nudges encouraging patients to get vaccinated at an upcoming doctor's appointment. Proc. Natl Acad. Sci. USA 118, e2101165118 (2021).

21. Milkman, K. L., Beshears, J., Choi, J. J., Laibson, D. \& Madrian, B. C. Using implementation intentions prompts to enhance influenza vaccination rates. Proc. Natl Acad. Sci. USA 108 10415-10420 (2011).

22. Sunstein, C. R. in The Handbook of Privacy Studies (eds Sloot, B. \& Groot, A.) 173-180 (Amsterdam Univ. Press, 2018).

23. Fishbane, A., Ouss, A. \& Shah, A. K. Behavioral nudges reduce failure to appear for court. Science 370, eabb6591 (2020).

24. Dale, A. \& Strauss, A. Don't forget to vote: text message reminders as a mobilization tool. Am. J. Pol. Sci. 53, 787-804 (2009).

25. Benartzi, S. et al. Should governments invest more in nudging? Psychol. Sci. 28, 1041-1055 (2017).

26. Loewenstein, G. \& Chater, N. Putting nudges in perspective. Behav. Public Policy 1, 26-53 (2017).

27. Pink, S. L., Chu, J., Druckman, J., Rand, D. G. \& Willer, R. Elite party cues increase vaccination intentions among Republicans. Proc. Natl Acad. Sci. USA 118, e2106559118 (2021).

28. Serra-Garcia, M. \& Szech, N. Choice architecture and incentives increase COVID-19 vaccine intentions and test demand. SSRN Electron J. CESifo Working Paper No. 9003 https://ssrn.com/abstract=3827616 (2021).

29. Pierce, J. L., Kostova, T. \& Dirks, K. T. Toward a theory of psychological ownership in organizations. Acad. Manage. Rev. 26, 298-310 (2001).

30. Shu, S. B. \& Peck, J. Psychological ownership and affective reaction: emotional attachment process variables and the endowment effect. J. Consum. Psychol. 21, 439-452 (2011).
31. Kreps, S. et al. Factors associated with US adults' likelihood of accepting COVID-19 vaccination. JAMA Netw. Open 3, e2O25594 (2020).

32. Lazarus, J. V. et al. A global survey of potential acceptance of a COVID-19 vaccine. Nat. Med. 27, 225-228 (2021).

33. Gomila, R. Logistic or linear? Estimating causal effects of experimental treatments on binary outcomes using regression analysis. J. Exp. Psychol. Gen. 150, 700-709 (2021).

34. Castillo, J. C. et al. Market design to accelerate COVID-19 vaccine supply. Science $\mathbf{3 7 1}$ 1107-1109 (2021).

35. Price-Haywood, E. G., Burton, J., Fort, D. \& Seoane, L. Hospitalization and mortality among black patients and white patients with COVID-19. N. Engl. J. Med. 382, 2534-2543 (2020).

36. Ruiz, J. B. \& Bell, R. A. Predictors of intention to vaccinate against COVID-19: Results of a nationwide survey. Vaccine 39, 1080-1086 (2021).

37. Holm, S. A simple sequentially rejective multiple test procedure. Scand. Stat. Theory Appl. 6, 65-70 (1979).

38. Fridman, A., Gershon, R. \& Gneezy, A. COVID-19 and vaccine hesitancy: a longitudinal study. PLoS ONE https://doi.org/10.1371/journal.pone.0250123 (2021).

39. Golman, R., Hagmann, D. \& Loewenstein, G. Information avoidance. J. Econ. Lit. 55, 96-135 (2017).

40. US Census Bureau. American Community Survey (ACS), https://www.census.gov/ programs-surveys/acs (accessed on 25 May 2021) (2020).

41. Funk, C. \& Tyson, A. Intent to get a COVID- 19 vaccine rises to $60 \%$ as confidence in research and development process increases. https://www.pewresearch.org/ science/2020/12/03/intent-to-get-a-covid-19-vaccine-rises-to-60-as-confidence-inresearch-and-development-process-increases/ (2020).

Publisher's note Springer Nature remains neutral with regard to jurisdictional claims in published maps and institutional affiliations.

Open Access This article is licensed under a Creative Commons Attribution 4.0 International License, which permits use, sharing, adaptation, distribution and reproduction in any medium or format, as long as you give appropriate credit to the original author(s) and the source, provide a link to the Creative Commons license, and indicate if changes were made. The images or other third party material in this article are included in the article's Creative Commons license, unless indicated otherwise in a credit line to the material. If material is not included in the article's Creative Commons license and your intended use is not permitted by statutory regulation or exceeds the permitted use, you will need to obtain permission directly from the copyright holder. To view a copy of this license, visit http://creativecommons.org/licenses/by/4.0/.

(c) The Author(s) 2021 


\section{Methods}

For RCTs, we predetermined the end date of enrolment for analyses reported herein, but we could not predetermine sample size by the enrolment deadline owing to uncertainty about how many UCLA Health participants would satisfy inclusion and exclusion criteria. We preregistered data-analysis plans contingent on the actual sample size on the basis of power analysis. We used power analysis to predetermine sample sizes for online experiments. RCTs and online experiments were randomized, and investigators were blinded to allocation during experiments.

\section{Ethics approval}

This research was deemed to comply with all relevant ethical regulations. The Institutional Review Board at the UCLA approved the protocols of our randomized controlled trials (reference number 21-000268) and determined that a waiver of informed consent was appropriate. All online experiments and the vaccination intention survey were conducted under approval of the Institutional Review Board at Carnegie Mellon University (reference number IRBSTUDY2015_00000482), and informed consent was obtained from all online study participants as part of the enrolment process.

\section{Setting for the RCTs}

We conducted the RCTs in partnership with UCLA Health, a large integrated academic health system in California. Extended Data Table 7 provides a comparison of demographic characteristics and vaccination rates between our RCT sample, UCLA Health primary and specialty care attributed patient population, Los Angeles County and California.

\section{Enrolment and eligibility for RCTs}

Starting from 19 January 2021, UCLA Health invited primary and speciality care attributed patients who were eligible for the COVID-19 vaccine at the time to get vaccinated. UCLA Health followed the national Advisory Committee on Immunization Practices as well as state and county guidelines to determine patient COVID-19 vaccine eligibility phasing. Considering the large volumes of eligible patients in each phase, UCLA Health developed a risk model that incorporates clinical and social risk to subprioritize within each phase. According to this model, UCLA Health sent invitations to eligible patients in batches over time to guarantee enough vaccine supply for invited patients. The size of the batch was decided daily on the basis of (1) available doses, (2) available appointment slots and (3) expected appointment rate. If UCLA Health identified a patient as having already obtained the vaccine inside or outside UCLA Health when it was their turn to be invited, the health system did not send the invitation to that patient.

On the first reminder date, patients were automatically enrolled into the first RCT and became participants if they (1) had a SMS-capable telephone number, (2) had not scheduled the first-dose COVID-19 vaccination appointment at UCLA Health and (3) had not obtained the first dose anywhere by the end of the day before the first reminder date, according to the latest California Immunization Registry (CAIR) records UCLA Health could access as well as UCLA Health's internal records. The earliest first reminder date was 1 February 2021.

On the second reminder date, patients were automatically enrolled in the second RCT and became participants if they (1) had a SMS-capable telephone number, (2) had not scheduled the first-dose COVID-19 vaccination appointment at UCLA Health and (3) had not obtained the first dose anywhere by the end of the day before the second reminder date. The earliest second reminder date was 9 February 2021.

Figure 1 shows the timeline, eligibility and randomization of the two RCTs. For both RCTs, participants within each batch were randomized at the individual level to treatments according to the design detailed in 'Design of the first-reminder RCT' and 'Design of the second-reminder RCT'. Enrolment was conducted by the UCLA Health Office of Population
Health and Accountable Care. Random assignment to interventions was performed by UCLA Health statisticians blind to the hypotheses and interventions using a computerized random number generator.

\section{Design of the first-reminder RCT}

We randomly assigned participants following simple randomized procedures at a 4:1 ratio to either the follow-through reminder arm, in which they received a reminder at 15:00 $\mathrm{h}$ on the first reminder date, or the holdout arm, in which they received no reminders. All reminders were designed to nudge individuals to schedule their vaccination appointments by (1) making vaccination top of mind to curb forgetfulness, and (2) providing the direct link to the scheduling website to reduce friction and increase convenience. The basic reminder read 'UCLA Health: [participant's first name], you can get the COVID-19 vaccine at UCLA Health. Make a vaccination appointment here: uclahealth. org/schedule.'

We nested a $2 \times 2$ factorial design within the follow-through reminder arm. The first factor was whether or not the reminder sought to enhance participants' feelings of psychological ownership over the vaccine to amplify their desire to obtain their vaccine (ownership intervention). The ownership intervention added language to the reminder to make participants feel as if the vaccine was already theirs. The ownership reminder read 'UCLA Health: [participant's first name], a COVID-19 vaccine has just been made available to you at UCLA Health. Claim your dose today by making a vaccination appointment here: uclahealth. org/schedule.'

The second factor was whether or not the reminder linked to a video that was designed to shift vaccination intentions by providing information about COVID-19 and the authorized vaccines (video intervention). The video intervention was based on a survey of the vaccine hesitancy literature ${ }^{3,31,32,36}$ as well as a survey that we conducted in January 2021 with California residents (as described in 'Vaccination intention survey'). The video (Supplementary Video 1) first highlighted the pandemic as a challenge, providing statistics on infections and ease of transmission. It then proposed the vaccine as an easy and safe solution, providing information about its effectiveness. The basic reminder with video read 'UCLA Health: [participant's first name], you can get the COVID-19 vaccine at UCLA Health. Please watch this important 2 min video: [link]. Make a vaccination appointment here: uclahealth.org/schedule.'

In the ownership reminder with video sub-arm, the reminder contained both the ownership and video interventions and read: 'UCLA Health: [participant's first name], a COVID-19 vaccine has just been made available to you at UCLA Health. Please take 2 simple steps: 1. Watch this important 2 min video: [link]. 2. Claim your dose today by making a vaccination appointment here: uclahealth.org/schedule.'

In all sub-arms, participants whose preferred language was Spanish received the text reminder (and the video (Supplementary Video 2), in the relevant cases) in Spanish. Participants within the follow-through reminder arm were randomly assigned following simple randomization procedures to one of these four sub-arms with an equal probability.

\section{Design of the second-reminder RCT}

Eight days after the initial notification, eligible participants were enrolled in the second RCT. They were randomized following simple randomization procedures at a 6:1 ratio to the follow-through reminder arm, in which another text reminder was sent at 15:00 h on the second reminder date, or the holdout arm with no reminders. Randomization was independent between the first and second RCTs (Supplementary Information section 1.1). Similar to the first RCT, all text reminders in the second RCT heightened the salience of vaccine availability (so as to combat forgetfulness) and provided the direct link to the appointment scheduling website (so as to increase convenience).

We nested a $2 \times 3$ factorial design within the follow-through reminder arm, in which we leveraged behavioural insights to motivate people to schedule a vaccination appointment via different messaging. 
The first factor varied whether the reminder emphasized prosocial (versus personal) benefits of getting vaccinated ${ }^{42,43}$. The second factor manipulated whether the reminder highlighted the exclusivity of having early access to the vaccine (early access framing), whether it framed the act of obtaining the vaccine as an opportunity to chart a new path forward (fresh start framing) or neither. The early access framing sought to leverage the principle of scarcity to increase vaccine demand ${ }^{44,45}$, as vaccination was still exclusive at the early stage of distribution (January-February 2021). The fresh start framing was inspired by previous work showing that people are motivated to take actions at new beginnings $s^{46,47}$. Here, we tested whether framing getting the vaccine as an opportunity to chart a new path forward for participants themselves or society could mobilize participants to get inoculated.

Specifically, the basic self/prosocial reminders read 'UCLA Health: [participant's name], to protect (yourself/your family, friends, and community), make your COVID-19 vaccine appointment here today: uclahealth.org/schedule." The early access self/prosocial reminders read 'UCLA Health: [participant's name], you are one of few Americans who have early access to the COVID-19 vaccine based on national guidelines. Take this opportunity to protect (yourself/your family, friends, and community who may not have this access yet). Make your vaccine appointment here today: uclahealth.org/schedule.' The fresh start self/ prosocial reminder read 'UCLA Health: [participant's name], (the past year has been tough/the past year has been tough for many). Now, the COVID-19 vaccine can offer the promise of a fresh start. Take this opportunity to protect (yourself/your family, friends, and community) and (chart a new path forward/help our nation chart a new path forward). Make your vaccine appointment here today: uclahealth.org/schedule.' The content in parentheses differed between the personal and prosocial messaging conditions. Participants within the follow-through reminder arm were randomly assigned following simple randomization procedures to one of these six sub-arms with an equal probability.

\section{Analyses and exclusion criteria of RCTs}

All analyses and exclusion criteria follow the preregistrations. We focus on participants enrolled in either RCT by 23 February 2021. This sample consists of participants eligible to get vaccinated at UCLA Health from 19 January to 22 February 2021, including participants at or above 65 years old, participants with any transplant and high-risk participants with qualifying pre-existing conditions. We report results using data extracted on 25 May 2021. We excluded participants who were enrolled in the first (second) RCT but either scheduled a vaccination appointment at UCLA Health by 15:00 $\mathrm{h}$ on their corresponding first (second) reminder date or obtained a COVID-19 vaccine somewhere before their corresponding first (second) reminder date according to the latest appointment and vaccination records UCLA Health could access on 25 May 2021. These participants could not have been motivated to schedule or obtain the first dose by our text reminders; thus, excluding them allows us to more accurately estimate the effect of our interventions on participants who could benefit from receiving our interventions. We additionally excluded participants under 18 years old as we only applied for the permission of the Institutional Review Board to analyse data about adult participants. The proportion of participants who were excluded in the analysis stage did not statistically significantly differ across conditions (Supplementary Table 1), and our results are robust if we conduct intent-to-treat analyses involving all participants who were enrolled in the RCTs by 23 February 2021 (Supplementary Information section 1.5).

For the first RCT, our preregistered analysis about participants enrolled by 23 February 2021 aimed to investigate (1) the average effect of sending a follow-through reminder; (2) whether all reminder types would outperform the holdout arm; (3) the effect of adding the video intervention to the reminder; (4) the effect of adding the ownership intervention; and (5) whether the aforementioned effects would differ between participants who received versus did not receive the influenza vaccine in either of two recent influenza seasons.

For the second RCT, our preregistered analysis about participants enrolled by 23 February 2021 aimed to investigate (1) the average effect of sending a second follow-through reminder and (2) whether all reminder types outperformed the holdout arm. Because we were uncertain about how many people would be enrolled in the second RCT by 23 February 2021, we preregistered to not compare sub-arms to each other with this data. Supplementary Information sections 1.3 and 1.4 describe for the scope of analyses we plan to conduct once the full data collection has been completed about all participants ever enrolled in our RCTs from the beginning of the trials until UCLA Health stops sending out COVID-19 vaccine invitations.

\section{Outcome measures for RCTs}

Our preregistered primary outcome measure indicates whether participants scheduled a vaccination appointment for the first dose of COVID-19 vaccine at UCLA Health within six days of the first (second) reminder date (specifically, from 15:00 h on the first (second) reminder date to 23:59 $\mathrm{h}$ on the fifth day following the first (second) reminder date.). We preregistered this time window because UCLA Health targeted additional outreach efforts to participants who had not scheduled their vaccination appointment six days after the second reminder date and we wanted to use a consistent time window for the two RCTs. The results are robust to extending the time window to one month (Supplementary Tables 22 and 23). Our secondary outcome measure in this Article is whether participants obtained the first dose of COVID-19 vaccine at UCLA Health within four weeks of the first (second) reminder date. We chose this window because UCLA Health generally only allowed participants to schedule an appointment for less than four weeks ahead. Consistent with this practice, $96.25 \%$ of the first-dose appointments made by participants in the analysis sample of the first RCT occurred within four weeks from the day they were scheduled. In the preregistrations, we listed additional secondary outcome variables; we explain why we did not focus on these in this Article in Supplementary Information section 1.2.

\section{Procedures for online experiments}

We ran two preregistered online experiments in February 2021, concurrently with the randomized controlled trials. In addition, we ran a preregistered replication experiment online in April 2021, when all US adults had become eligible to receive the vaccine.

In the February 2021 experiments, we instructed participants to imagine becoming eligible for the COVID-19 vaccine and receiving a text message from their healthcare provider encouraging them to get vaccinated. We randomly assigned participants to read one of the four reminders from the first RCT. Participants in the 'video' conditions were also instructed to watch the video. After reading the message, participants indicated their likelihood of scheduling a vaccination appointment: 'How likely would you be to schedule a vaccination appointment after receiving this message from your healthcare provider?' (1, not at all likely, to 7, very likely). They also rated the persuasiveness of the text message ( 1 , not at all persuasive, to 7 , very persuasive). To check whether the messages containing ownership language increased feelings of psychological ownership over the vaccine as we intended, we asked participants, 'To what extent does the text message make you feel that the COVID-19 vaccine is already yours?' (1, not at all, to 7, very much $)^{30}$. To understand how the video may have changed viewers' perceptions and beliefs, we measured participants' beliefs about the prevalence of COVID-19, worry about spreading the virus, perceived vaccine effectiveness, anticipated regret for not getting the vaccine and trust in the vaccine (Supplementary Information section 2 for questions and results).

The April 2021 experiment used identical procedures but adopted additional measures of vaccination intentions to test whether findings 
in our February 2021 studies are robust to different ways of soliciting intentions. For this purpose, we randomized whether participants answered questions in the same hypothetical manner as in the February studies, or responded to questions with a less hypothetical framing. Participants randomized to answer the hypothetical version were asked 'How likely would you be to schedule a vaccination appointment after receiving this message from your healthcare provider?' (1, not at all likely, to 7, very likely) and 'How much would you want the vaccine after receiving this message from your healthcare provider?' (1, not at all, to 7, very much). These two questions were highly correlated $(r=0.94$, $P<0.001)$ and aggregated into a composite. Participants randomized to answer the less hypothetical version of the intention questions were asked 'How likely are you to schedule a vaccination appointment today after receiving this message from your healthcare provider?' (1, not at all likely, to 7, very likely) and 'How much do you want the vaccine now, after receiving this message from your healthcare provider?' (1, not at all, to 7, very much). These two measures were highly correlated $(r=0.93$, $P<0.001)$ and averaged into a composite. All participants also rated the persuasiveness of the message they read, using the same measure used in the February studies (Supplementary Information section 3).

\section{Sample for online experiments}

We recruited participants from Amazon's Mechanical Turk (MTurk) and Prolific Academic (Prolific) who had not received a COVID-19 vaccine or scheduled a first-dose vaccination appointment at the time of the study. To be assigned to treatment, participants had to first pass a Captcha and an attention check question. To be included in the analysis, participants had to complete our preregistered dependent variables and not report having technical problems with the video. Considering these criteria, our first February 2021 online experiment consists of 1,163 participants. Our second February 2021 online experiment consists of 840 participants recruited from Prolific who satisfied similar criteria as those in the first online experiment, except that we additionally required that they did not report having taken a similar survey on MTurk. In both experiments, we attempted to recruit a balanced sample of individuals who self-reported as Democrat or Republican to test the generalizability of our findings (Supplementary Information section 2.1 for recruitment detail). Participants received US $\$ 0.90$ on MTurk and US $\$ 1.10$ on Prolific for completing our 6-min survey. Across the two February online experiments, our sample consists of 2,003 participants (47.1\% male, $71.8 \%$ white (excluding Hispanic or Latino), $51.8 \%$ Democrat, average age $=37.9$, s.d. $=13.4$ ).

For our April 2021 online experiment, we recruited participants on MTurk and Prolific using the same eligibility criteria as the second online experiment. Participants on MTurk received US $\$ 0.90$ or US $\$ 1.00$ (we boosted the pay to US $\$ 1.00$ on the third day of data collection to attract more respondents) and those on Prolific received US $\$ 1.10$ for completing our 6-min survey. Our sample consists of 1,178 participants (44.9\% male, $71.6 \%$ white (excluding Hispanic or Latino), $40.8 \%$ Democrat, average age $=36.7$, s.d. $=12.0$ ).

\section{Vaccination intention survey}

To design the video used in our first RCT, we ran a survey in January 2021 involving 515 residents of California recruited on MTurk and Prolific (49.3\% male, $42.9 \%$ white (excluding Hispanic or Latino), 70.9\% Democrat, average age $=33.9$, s.d. $=12.7)$. Participants received US $\$ 1.00$ on MTurk or US $\$ 1.20$ on Prolific for completing our 9-min survey. We asked participants to consider the authorized vaccines (Pfizer and Moderna) when taking the survey. We elicited their vaccination intentions by asking If one of the COVID-19 vaccines were available to you today, would you get the vaccine ${ }^{\prime 41}$. Participants chose one from four options: 'Definitely would get the vaccine', 'Probably would get the vaccine', 'Probably would not get the vaccine' and 'Definitely would not get the vaccine'. We then elicited participants' beliefs and perceptions about COVID-19 and the vaccines. Specifically, we measured beliefs about infection likelihood with and without the vaccine and the severity of COVID-19. We collected feelings of vulnerability to COVID-19, fear of infection, worry of transmitting COVID-19 to others, anticipated regret for not getting the vaccine and trust in the vaccine. We compared answers to these questions among people who reported that they definitely would get the vaccine versus those feeling more uncertain. Supplementary Information section 5 describes all variables and results.

\section{Methods of investigating intentions versus actual uptake}

In Extended Data Table 6, we report statistics about the estimated effects of adding ownership language and a video-based information intervention to a reminder on vaccination intentions (based on online experiments) versus actual vaccine uptake (based on the first RCT). The statistics we report include the $95 \%$ confidence interval, the absolute value of Cohen's $d$ or $h$, and $\eta_{p}{ }^{2}$ of each estimated effect. To calculate Cohen's $h$ for the binary outcomes measured in the first RCT, we use $2 \times \operatorname{arcsine} \sqrt{ } P_{\text {with an intervention }}-2 \times \operatorname{arcsine} \sqrt{ } P_{\text {without an intervention }}{ }^{48}$ in which $\sqrt{ } P_{\text {with an intervention }}$ represents the percentage of participants who scheduled an appointment for (or obtained) the first dose at UCLA Health within six days (or within four weeks) of the first reminder date among those who received a text reminder containing a given intervention and $\sqrt{ } P_{\text {without an intervention }}$ represents the percentage among participants who received a text reminder without that intervention. To calculate $\eta_{\mathrm{p}}{ }^{2}$ for the online experiments and the first RCT, we use $\eta_{\mathrm{p}}{ }^{2}=F \times d f_{\text {numerator }} /\left(F \times d f_{\text {numerator }}+d f_{\text {denominator }}\right)^{49}$ in which the $F$ value and numerator and denominator degrees of freedom came from the OLS regressions reported in Supplementary Tables 5, 39.

\section{Reporting summary}

Further information on research design is available in the Nature Research Reporting Summary linked to this paper.

\section{Data availability}

The two RCTs were pre-registered at clinicaltrials.gov (first-reminder RCT, https://clinicaltrials.gov/ct2/show/NCT04800965; second-reminder RCT, https://clinicaltrials.gov/ct2/show/ NCT04801524). The three online experiments were preregistered at aspredicted.org (online experiment 1, https://aspredicted.org/ blind.php? $\mathrm{x}=\mathrm{u} 2 \mathrm{ng} 5 \mathrm{c}$; online experiment 2 , https://aspredicted. org/blind.php? $x=a e 3 c i 5$; and online experiment 3, https://aspredicted.org/blind.php? $\mathrm{x}=7 \mathrm{wf}$ 9er and https://aspredicted.org/blind. php? $\mathrm{x}=\mathrm{u} 82 \mathrm{hy} 5)$. The data analysed in this Article about randomized controlled trials were provided by UCLA Health and contain protected health information. To protect participant privacy, we cannot publicly post individual-level data. Qualified researchers with a valuable research question and relevant approvals including ethical approval can request access to the de-identified data about these trials from the corresponding author. A formal contract will be signed and an independent data protection agency should oversee the sharing process to ensure the safety of the data. Data about our online experiments and vaccination intention survey are available at: https://osf.io/ qn8hr/?view_only=cf7b2bc590054aee8c4a2bae99ef20c5.

\section{Code availability}

The code to replicate the analyses and figures in the Article and its Supplementary Information is available at https://osf.io/qn8hr/?view_onl $\mathrm{y}=\mathrm{cf7}$ b2bc590054aee8c4a2bae99ef20c5.

42. Li, M., Taylor, E. G., Atkins, K. E., Chapman, G. B. \& Galvani, A. P. Stimulating influenza vaccination via prosocial motives. PLoS ONE 11, e0159780 (2016).

43. Grant, A. M. \& Hofmann, D. A. It's not all about me: motivating hand hygiene among health care professionals by focusing on patients. Psychol. Sci. 22, 1494-1499 (2011).

44. Goldstein, N. J., Martin, S. J. \& Cialdini, R. B. Yes!: 50 Scientifically Proven Ways to Be Persuasive (Free, 2008) 
45. Imas, A. \& Madarasz, K. Mimetic dominance and the economics of exclusion: private goods in public context. SSRN Electron J. https://doi.org/10.2139/ssrn.3630697 (2020)

46. Dai, H., Milkman, K. L. \& Riis, J. The fresh start effect: temporal landmarks motivate aspirational behavior. Manage. Sci. 60, 2563-2582 (2014).

47. Dai, H., Milkman, K. L. \& Riis, J. Put your imperfections behind you: temporal landmarks spur goal initiation when they signal new beginnings. Psychol. Sci. 26, 1927-1936 (2015).

48. Cohen, J. Statistical Power Analysis for the Behavioral Sciences (2nd ed.) (Lawrence Erlbaum Associates,1988)

49. Ben-Shachar, M., Lüdecke, D. \& Makowski, D. Effect size: estimation of effect size indices and standardized parameters. J. Open Source Softw. 5, 2815 (2020).

Acknowledgements Funding support for this research was provided by UCLA Health, Anderson School of Management, Anderson Behavioral Lab and Carnegie Mellon University. We thank UCLA Health, particularly L. Ho, J. Ileto, A. Machalinski, and T. Tieu, for making this research possible; S. Bhargava, K. Brabaw, G. Chapman, N. Goldstein, K. Haggag, I. Hurst,

C. Nyungen, K. Milkman, M. Serra-Garcia and K. Shonk for feedback on the paper; and

J. Cervantez, M. Lanyon and S. Permut for assistance with this research.
Author contributions D.M.C., H.D. and S.S. conceptualized the project; H.D., H.M., S.P., M.S and S.S. curated the data; H.D., S.S. and S.V. undertook formal analyses; D.M.C. and M.A.H. acquired funding; D.M.C., H.D., S.S., M.A.H., N.R., and L.R. performed the investigations; D.M.C., H.D., S.S. and M.A.H. designed the methodology; D.M.C., H.D. and S.S. administrated the project; D.M.C., H.D. and S.S. supervised the work; H.D., S.S. and S.V. validated the findings; H.D. and S.S. visualized the data; H.D. and S.S. wrote the original draft, and D.M.C., H.D., S.S., M.A.H., H.M., S.P., M.S., L.R., N.R., and S.V. contributed to the review and editing of the paper.

Competing interests The authors declare no competing interests. The authors did not receive financial or non-financial benefits from UCLA Health or speaking/consulting fees related to any of the interventions presented here.

\section{Additional information}

Supplementary information The online version contains supplementary material available at https://doi.org/10.1038/s41586-021-03843-2.

Correspondence and requests for materials should be addressed to D.M.C.

Peer review information Nature thanks the anonymous reviewers for their contribution to the peer review of this work.

Reprints and permissions information is available at http://www.nature.com/reprints. 
Article

Extended Data Table 1 | Randomization check of the first RCT

\begin{tabular}{|c|c|c|c|c|c|c|}
\hline & $\begin{array}{c}\text { Basic } \\
\text { Reminder }\end{array}$ & $\begin{array}{l}\text { Ownership } \\
\text { Reminder }\end{array}$ & $\begin{array}{c}\text { Basic Reminder } \\
\text { w/Video }\end{array}$ & $\begin{array}{c}\text { Ownership Reminder } \\
\text { w/Video }\end{array}$ & Holdout & $\begin{array}{l}F \text {-test } \\
\text { p-value }\end{array}$ \\
\hline Age (years) & $\begin{array}{c}72.7 \\
(10.3)\end{array}$ & $\begin{array}{c}72.8 \\
(10.4)\end{array}$ & $\begin{array}{c}72.8 \\
(10.3)\end{array}$ & $\begin{array}{c}72.8 \\
(10.4)\end{array}$ & $\begin{array}{c}73.0 \\
(10.2)\end{array}$ & 0.177 \\
\hline Male & $\begin{array}{c}43.3 \% \\
(49.6 \%)\end{array}$ & $\begin{array}{c}43.4 \% \\
(49.6 \%)\end{array}$ & $\begin{array}{c}43.5 \% \\
(49.6 \%)\end{array}$ & $\begin{array}{c}42.7 \% \\
(49.5 \%)\end{array}$ & $\begin{array}{c}43.5 \% \\
(49.6 \%)\end{array}$ & 0.497 \\
\hline White & $\begin{array}{c}53.2 \% \\
(49.9 \%)\end{array}$ & $\begin{array}{c}53.8 \% \\
(49.9 \%)\end{array}$ & $\begin{array}{l}52.8 \% \\
(49.9 \%)\end{array}$ & $\begin{array}{c}53.7 \% \\
(49.9 \%)\end{array}$ & $\begin{array}{c}53.9 \% \\
(49.9 \%)\end{array}$ & 0.165 \\
\hline Preferring Spanish & $\begin{array}{c}4.9 \% \\
(21.5 \%)\end{array}$ & $\begin{array}{c}5.1 \% \\
(22.0 \%)\end{array}$ & $\begin{array}{c}5.1 \% \\
(22.0 \%)\end{array}$ & $\begin{array}{c}4.8 \% \\
(21.4 \%)\end{array}$ & $\begin{array}{c}4.9 \% \\
(21.5 \%)\end{array}$ & 0.521 \\
\hline $\begin{array}{l}\text { Received Flu Shot } \\
\text { in Two Recent Seasons }\end{array}$ & $\begin{array}{c}49.8 \% \\
(50.0 \%)\end{array}$ & $\begin{array}{c}50.0 \% \\
(50.0 \%) \\
\end{array}$ & $\begin{array}{c}50.1 \% \\
(50.0 \%) \\
\end{array}$ & $\begin{array}{c}50.3 \% \\
(50.0 \%) \\
\end{array}$ & $\begin{array}{c}50.2 \% \\
(50.0 \%)\end{array}$ & 0.905 \\
\hline Number of Patients & 18,629 & 18,592 & 18,757 & 18,627 & 18,749 & \\
\hline
\end{tabular}

Average and s.d. (in parentheses) of participant demographic variables in each condition of the first RCT, the $P$ value of a two-sided $F$-test for joint significance across five conditions for each

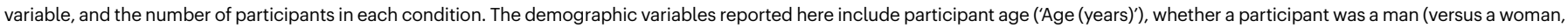
other or unknown; 'Male'), whether a participant was white (excluding Hispanic or Latino; 'white'), whether Spanish was a participant's preferred language ('Preferring Spanish'), and whether

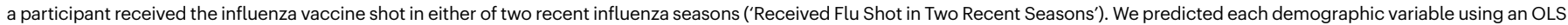

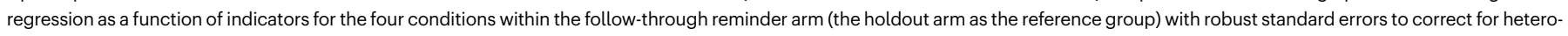

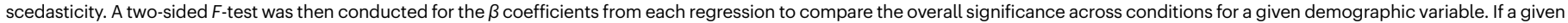
$P$ value is greater than 0.05 , it means the corresponding $F$-test does not allow us to reject the null hypothesis that all five conditions in the first RCT have the same value for the corresponding demographic variable. 
Extended Data Table 2 | Regression-estimated effects of text reminders on appointments and vaccinations at UCLA Health in the first RCT

\begin{tabular}{|c|c|c|c|c|c|c|c|}
\hline & \multicolumn{3}{|c|}{$\begin{array}{l}\text { Appointment at UCLA Health } \\
\text { (Within Six Days) }\end{array}$} & \multicolumn{3}{|c|}{$\begin{array}{l}\text { Vaccination at UCLA Health } \\
\text { (Within Four Weeks) }\end{array}$} & $\mathrm{N}$ \\
\hline \multicolumn{8}{|l|}{ Effects of Receiving a Text Reminder } \\
\hline Follow-Through Reminder (for White Patients) & 0.063 & 0.003 & $<0.001$ & 0.040 & 0.004 & $<0.001$ & 49,909 \\
\hline Follow-Through Reminder (for Hispanic Patients) & 0.059 & 0.007 & $<0.001$ & 0.033 & 0.009 & $<0.001$ & 10,624 \\
\hline Follow-Through Reminder (for Black Patients) & 0.084 & 0.011 & $<0.001$ & 0.048 & 0.014 & 0.001 & 5,109 \\
\hline Basic Reminder & 0.055 & 0.003 & $<0.001$ & 0.031 & 0.004 & $<0.001$ & 37,378 \\
\hline Reminders Containing Ownership Language & 0.068 & 0.003 & $<0.001$ & 0.041 & 0.003 & $<0.001$ & 55,968 \\
\hline Remnders Containing the Video & 0.059 & 0.003 & $<0.001$ & 0.035 & 0.003 & $<0.001$ & 56,133 \\
\hline \multicolumn{8}{|l|}{ Effects of Adding an Intervention to the Reminder } \\
\hline Ownership Framing & 0.015 & 0.002 & $<0.001$ & 0.011 & 0.003 & $<0.001$ & 74,605 \\
\hline
\end{tabular}

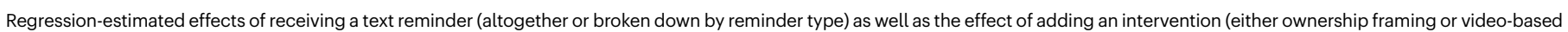
information intervention) to the reminder on appointments and vaccinations at UCLA Health in the first RCT. The two outcome measures are whether participants scheduled an appointment to get the first dose of the COVID-19 vaccine at UCLA Health from 15:00 h on the first reminder date to 23:59 h on the fifth day following the first reminder date ('Appointment at UCLA (Within Six Days)') and whether participants obtained the first dose of the COVID-19 vaccine at UCLA Health within four weeks of the first reminder date ('Vaccinated at UCLA (Within Four Weeks)').

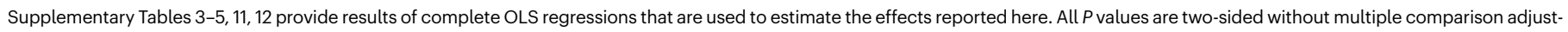

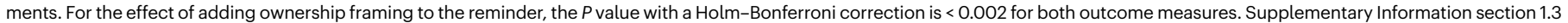
describes how we handle multiple comparisons. ' $N$ ' refers to the number of observations in each regression. 
Article

Extended Data Table 3 | Randomization check of the second RCT

\begin{tabular}{|c|c|c|c|c|c|c|c|c|}
\hline Age (years) & $\begin{array}{c}73.7 \\
(10.1)\end{array}$ & $\begin{array}{c}73.6 \\
(10.1)\end{array}$ & $\begin{array}{l}73.7 \\
(9.9)\end{array}$ & $\begin{array}{c}73.7 \\
(10.0)\end{array}$ & $\begin{array}{l}73.7 \\
(9.9)\end{array}$ & $\begin{array}{l}73.9 \\
(9.9)\end{array}$ & $\begin{array}{l}73.7 \\
(9.8)\end{array}$ & 0.389 \\
\hline Male & $\begin{array}{c}43.3 \% \\
(49.5 \%)\end{array}$ & $\begin{array}{c}43.4 \% \\
(49.6 \%)\end{array}$ & $\begin{array}{c}43.6 \% \\
(49.6 \%)\end{array}$ & $\begin{array}{c}44.1 \% \\
(49.7 \%)\end{array}$ & $\begin{array}{c}43.3 \% \\
(49.6 \%)\end{array}$ & $\begin{array}{c}42.8 \% \\
(49.5 \%)\end{array}$ & $\begin{array}{c}44.0 \% \\
(49.6 \%)\end{array}$ & 0.551 \\
\hline Preferring Spanish & $\begin{array}{c}5.5 \% \\
(22.8 \%)\end{array}$ & $\begin{array}{c}5.2 \% \\
(22.2 \%)\end{array}$ & $\begin{array}{c}5.2 \% \\
(22.3 \%)\end{array}$ & $\begin{array}{c}5.2 \% \\
(22.2 \%)\end{array}$ & $\begin{array}{c}4.8 \% \\
(21.4 \%)\end{array}$ & $\begin{array}{c}5.2 \% \\
(22.3 \%)\end{array}$ & $\begin{array}{c}5.5 \% \\
(22.8 \%)\end{array}$ & 0.402 \\
\hline $\begin{array}{l}\text { Received Flu Shot } \\
\text { in Two Recent Seasons }\end{array}$ & $\begin{array}{c}44.4 \% \\
(49.7 \%)\end{array}$ & $\begin{array}{c}44.9 \% \\
(49.7 \%)\end{array}$ & $\begin{array}{c}44.3 \% \\
(49.7 \%)\end{array}$ & $\begin{array}{c}45.0 \% \\
(49.7 \%)\end{array}$ & $\begin{array}{c}44.6 \% \\
(49.7 \%)\end{array}$ & $\begin{array}{c}44.7 \% \\
(49.7 \%)\end{array}$ & $\begin{array}{c}45.4 \% \\
(49.8 \%)\end{array}$ & 0.798 \\
\hline
\end{tabular}

Average and s.d. (in parentheses) of participant demographic variables in each condition of the second RCT, the $P$ value of a two-sided $F$-test for joint significance across seven conditions for each variable, and the number of participants in each condition. The demographic variables reported here include participant age ('Age (years)'), whether a participant was a man (versus a woman, other or unknown; 'Male'), whether a participant was white (excluding Hispanic or Latino; 'White'), whether Spanish was a participant's preferred language ('Preferring Spanish'), and whether a participant received the influenza vaccine in either of two recent influenza seasons ('Received Flu Shot in Two Recent Seasons'). We predicted each demographic variable using an OLS regression as a function of indicators for the six conditions within the follow-through reminder arm (the holdout arm as the reference group) with robust standard errors to correct for

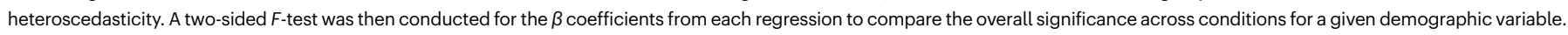

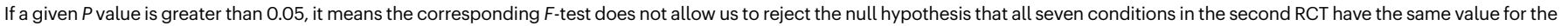
corresponding demographic variable. 
Extended Data Table 4 | Regression-estimated effects of text reminders on appointments and vaccinations at UCLA Health in the second RCT

\begin{tabular}{|c|c|c|c|c|c|c|c|}
\hline & \multicolumn{3}{|c|}{$\begin{array}{l}\text { Appointment at UCLA Health } \\
\text { (Within Six Days) }\end{array}$} & \multicolumn{3}{|c|}{$\begin{array}{l}\text { Vaccination at UCLA Health } \\
\text { (Within Four Weeks) }\end{array}$} & \multirow[b]{2}{*}{$\mathrm{N}$} \\
\hline & $\mathrm{B}$ & $\mathrm{SE}$ & p-value & $\mathrm{B}$ & SE & $p$-value & \\
\hline \multicolumn{8}{|l|}{ Effects of Receiving a Text Reminder } \\
\hline Follow-Through Reminder & 0.017 & 0.002 & $<0.001$ & 0.011 & 0.003 & $<0.001$ & 67,092 \\
\hline \multicolumn{8}{|l|}{ Effects of Receiving a Specific Type of Reminder } \\
\hline Reminders Highlighting Self Benefits & 0.015 & 0.002 & $<0.001$ & 0.009 & 0.003 & 0.003 & 38,406 \\
\hline Reminders Highlighting Prosocial Benefits & 0.018 & 0.002 & $<0.001$ & 0.013 & 0.003 & $<0.001$ & 38,311 \\
\hline Reminders Highlighting Exclusivity & 0.020 & 0.002 & $<0.001$ & 0.013 & 0.003 & $<0.001$ & 28,765 \\
\hline Reminders Highlighting Fresh Start Opportunities & 0.013 & 0.002 & $<0.001$ & 0.009 & 0.003 & 0.004 & 28,759 \\
\hline
\end{tabular}

Regression-estimated effects of receiving a text reminder (altogether or broken down by reminder type) on appointments and vaccinations at UCLA Health in the second RCT. The two outcome measures are whether participants scheduled an appointment to get the first dose of the COVID-19 vaccine at UCLA Health from 15:00 h on the second reminder date to $23: 59 \mathrm{~h}$ on the fifth day following the second reminder date ('Appointment at UCLA (Within Six Days)') and whether participants obtained the first dose of the COVID-19 vaccine at UCLA Health within four weeks of the second reminder date ('Vaccinated at UCLA (Within Four Weeks)'). Supplementary Tables 14, 15 provide results of complete OLS regressions that are used to estimate the effects reported here. All $P$ values are two-sided without multiple comparison adjustments. ' $\mathrm{N}$ ' refers to the number of observations in each regression. 


\section{Article}

Extended Data Table 5 | Regression-estimated effects of ownership framing and video-based information intervention in online experiments

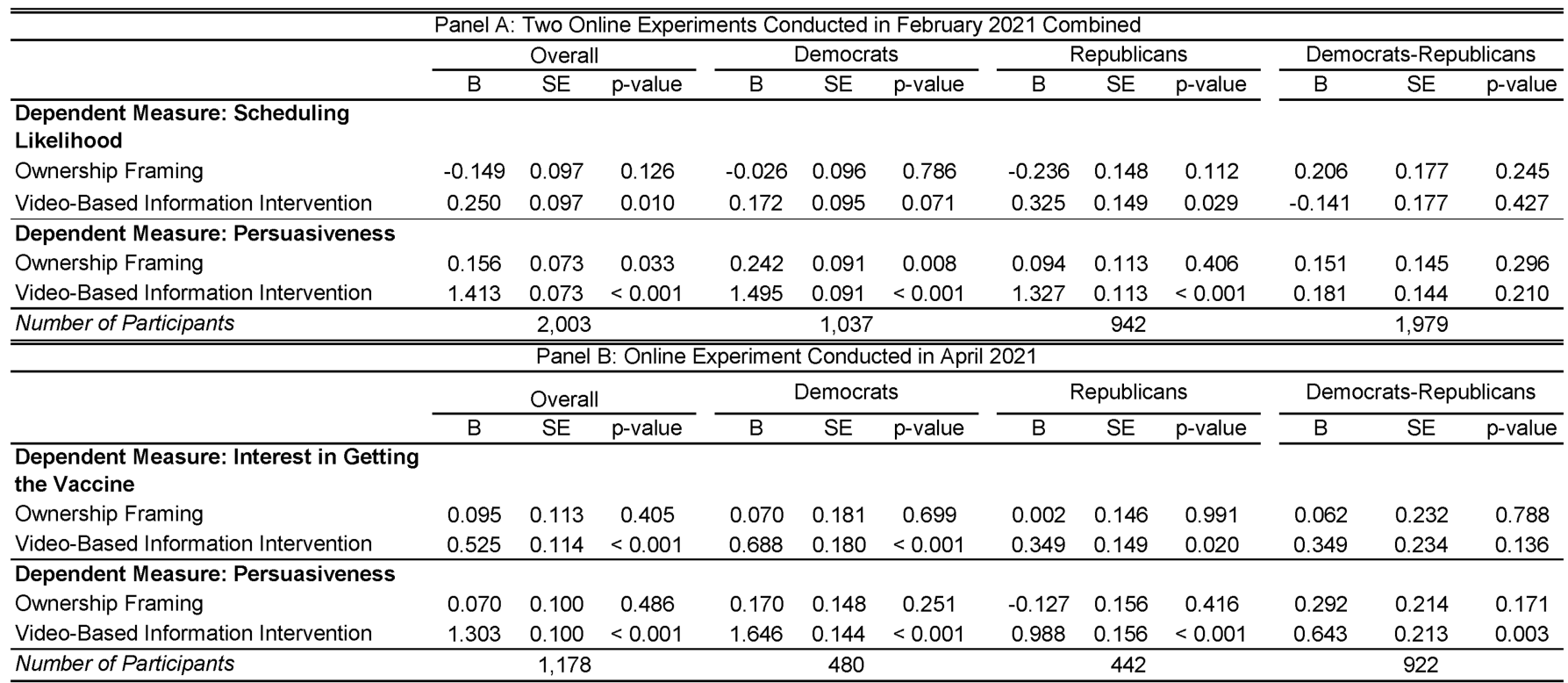

Regression-estimated effects of adding ownership framing and a video-based information intervention to a text reminder in online experiments, across all participants as well as broken down by political party. a, Combined results for the two online experiments conducted in February 2021. These experiments assessed participants' self-reported likelihood of scheduling an appointment for the COVID-19 vaccine after getting a text message from their healthcare provider ('Scheduling Likelihood') as well as how persuasive participants found the message to be ('Persuasiveness). b, Results for the online experiment conducted in April 2021. This experiment assessed participants' interest in getting the COVID-19 vaccine after getting a text message

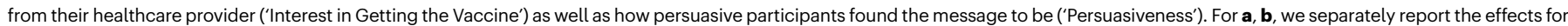

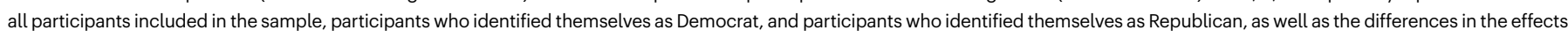
between Democrats and Republicans. Supplementary Tables $25,28,35,38$ provide results of complete OLS regressions that are used to estimate the effects reported here. All $P$ values are two-sided without multiple comparison adjustments. 


\section{Extended Data Table 6 | Comparison between the first RCT and online experiments}

\begin{tabular}{|c|c|c|}
\hline & Online Experiments Pooled & First RCT \\
\hline Population & US adults recruited from MTurk and Prolific & $\begin{array}{l}\text { UCLA Health adult patients, who are mostly California and } \\
\text { primarily Los Angeles residents }\end{array}$ \\
\hline Key Dependent Measures & $\begin{array}{l}\text { Likelihood of scheduling an appointment based } \\
\text { on self-report ( } 1=\text { Not likely at all; } 7=\text { Very } \\
\text { likely }) \text {; } \\
\text { The extent to which a participant wanted the } \\
\text { vaccine based on self-report }(1=\text { Not at all; } 7= \\
\text { Very much) }\end{array}$ & $\begin{array}{l}\text { A binary variable indicating whether a patient scheduled the } \\
\text { first-dose appointment at UCLA Health within six days based } \\
\text { on UCLA Health's records }(1=\text { Yes; } 0=\text { No); } \\
\text { A binary variable indicating whether a patient obtained the } \\
\text { first dose at UCLA Health within four weeks based on UCLA } \\
\text { Health's records }(1=\text { Yes; } 0=\mathrm{No})\end{array}$ \\
\hline$\%$ Male & $46.3 \%$ & $43.3 \%$ \\
\hline Mean of Age (SD) & $37.5(12.9)$ & $72.8(10.3)$ \\
\hline The Ownership Intervention & $\begin{array}{l}\mathrm{B}=-0.06(95 \% \mathrm{Cl}=[-0.22,0.10]) ; \text { Cohen's } d \\
=0.02 ; \eta_{p}^{2}=0.0002\end{array}$ & $\begin{array}{l}\text { For appointments at UCLA Health: } \mathrm{B}=0.015(95 \% \mathrm{Cl}= \\
[0.010,0.020]) \text {; Cohen's } h=0.046 ; \eta_{p}^{2}=0.0005 \\
\text { For vaccinations at UCLA Health: } \mathrm{B}=0.011(95 \% \mathrm{Cl}= \\
[0.006,0.016]) ; \text { Cohen's } h=0.031 ; \eta_{p}^{2}=0.0002\end{array}$ \\
\hline The Video Intervention & $\begin{array}{l}\mathrm{B}=0.38(95 \% \mathrm{Cl}=[0.22,0.54]) ; \text { Cohen's } d= \\
0.16 ; \eta_{p}^{2}=0.007\end{array}$ & $\begin{array}{l}\text { For appointments at UCLA Health: } \mathrm{B}=-0.003(95 \% \mathrm{Cl}= \\
[-0.008,0.002]) ; \text { Cohen's } h=0.007 ; \eta_{p}^{2}=0.00002 \\
\text { For vaccinations at UCLA Health: } \mathrm{B}=-0.002(95 \% \mathrm{Cl}= \\
[-0.007,0.003]) ; \text { Cohen's } h=0.004 ; \eta_{p}^{2}=6.1 * e^{-06}\end{array}$ \\
\hline Sample Size & 3,181 & $\begin{array}{l}93,354 \text { (including } 74,605 \text { patients in the Follow-Through Re- } \\
\text { minder arm) }\end{array}$ \\
\hline
\end{tabular}

Comparison of the first RCT and online experiments (pooled across three experiments) in their primary inclusion criteria, key dependent measures, sample characteristics (including the percentage of men, the average and s.d. of participant age, the percentage of participants who were white (excluding Hispanic or Latino), and the percentage of participants who received

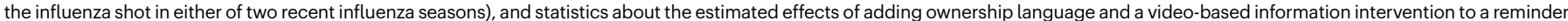

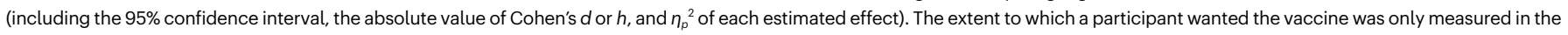
online experiment conducted in April 2021; for participants in this experiment, the outcome measure was the average of their responses to the two questions listed above. The methods for

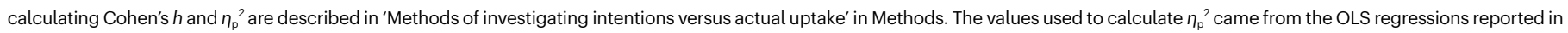
Supplementary Tables 5, 39. Supplementary Information section 4 provides a discussion about this table. 


\section{Article}

Extended Data Table 7 | Comparison between UCLA Health participants, California residents and Los Angeles County residents

\begin{tabular}{|c|c|c|c|c|c|}
\hline & $\begin{array}{c}\text { (1) } \\
\text { California } \\
\text { Residents }\end{array}$ & $\begin{array}{c}\text { (2) } \\
\text { LA County } \\
\text { Residents }\end{array}$ & $\begin{array}{c}\text { (3) } \\
\text { UCLA Health } \\
\text { Primary \& Specialty } \\
\text { Care Patients }\end{array}$ & $\begin{array}{l}\text { (4) } \\
\text { Patients Enrolled } \\
\text { in the First RCT } \\
\text { by } 2 / 23 / 2021\end{array}$ & $\begin{array}{l}\text { Patients in Column (4) Included } \\
\text { in the Analysis of the First RCT } \\
\text { (After Exclusion Criteria) }\end{array}$ \\
\hline$\%$ of Female & $50.3 \%$ & $50.7 \%$ & $56.6 \%$ & $56.8 \%$ & $56.7 \%$ \\
\hline$\%$ of Seniors $(\geq 65)$ & $14.8 \%$ & $14.1 \%$ & $25.6 \%$ & $90.8 \%$ & $90.1 \%$ \\
\hline$\%$ of White & $71.9 \%$ & $70.7 \%$ & $53.7 \%$ & $60.5 \%$ & $58.6 \%$ \\
\hline $\begin{array}{l}\text { \% of White Excluding } \\
\text { Unknown-Race Patients }\end{array}$ & $71.9 \%$ & $70.7 \%$ & $66.0 \%$ & $70.6 \%$ & $68.6 \%$ \\
\hline$\%$ of Hispanic or Latino & $39.4 \%$ & $48.6 \%$ & $12.1 \%$ & $10.1 \%$ & $11.4 \%$ \\
\hline $\begin{array}{l}\text { First-Dose Vaccination Rates } \\
\text { Among Seniors by } 2 / 23 / 2021\end{array}$ & $49 \%$ & $55 \%$ & $62 \%$ & $46 \%$ & $24 \%$ \\
\hline $\begin{array}{l}\text { First-Dose Vaccination Rates } \\
\text { Among Seniors by } 3 / 23 / 2021\end{array}$ & $67 \%$ & $72 \%$ & $72 \%$ & $58 \%$ & $40 \%$ \\
\hline $\begin{array}{l}\text { First-Dose Vaccination Rates } \\
\text { Among Seniors by } 4 / 23 / 2021\end{array}$ & $74 \%$ & $81 \%$ & $75 \%$ & $62 \%$ & $46 \%$ \\
\hline
\end{tabular}

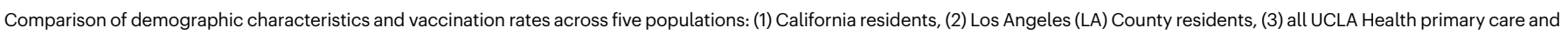

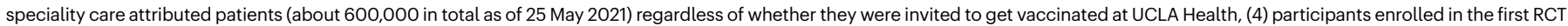
by 23 February 2021 ( $n=132,337$ ), and (5) participants in our analysis of the first RCT (that is, participants who were enrolled in the first RCT by 23 February 2021 and were not further excluded from analyses based on our exclusion criteria) $(n=93,354)$. Demographic data of California residents and Los Angeles County residents were accessed from https://www.census.gov/quick-

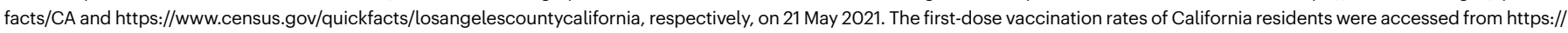
covid19.ca.gov/vaccination-progress-data/\\#progress-by-group on 21 May 2021. To calculate the first-dose vaccination rates of Los Angeles County residents, we divided the number of senior

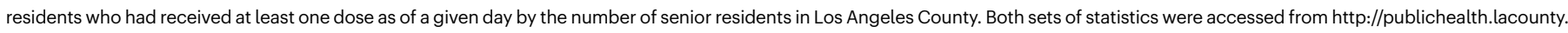

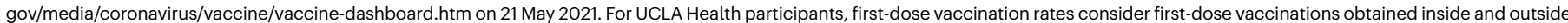
UCLA Health. '\% of White' refers to the percentage of white alone (excluding a 'mixed race' self-identification) for California and Los Angeles County residents but includes this 'mixed race'

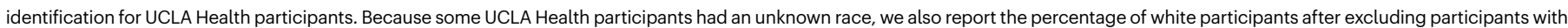
an unknown race, to be more comparable to California and Los Angeles Country statistics (which do not have an unknown race category). Supplementary Information section 1.6 provides a discussion about this table. 


\section{nature portfolio}

Corresponding author(s): Daniel Croymans

Last updated by author(s): 2021/7/5

\section{Reporting Summary}

Nature Portfolio wishes to improve the reproducibility of the work that we publish. This form provides structure for consistency and transparency in reporting. For further information on Nature Portfolio policies, see our Editorial Policies and the Editorial Policy Checklist.

\section{Statistics}

For all statistical analyses, confirm that the following items are present in the figure legend, table legend, main text, or Methods section.

n/a Confirmed

$\bigotimes$ The exact sample size $(n)$ for each experimental group/condition, given as a discrete number and unit of measurement

$\bigotimes$ A statement on whether measurements were taken from distinct samples or whether the same sample was measured repeatedly

$\triangle$ The statistical test(s) used AND whether they are one- or two-sided

Only common tests should be described solely by name; describe more complex techniques in the Methods section.

$\bigotimes$ A description of all covariates tested

$\bigotimes$ A description of any assumptions or corrections, such as tests of normality and adjustment for multiple comparisons

$\triangle$ A full description of the statistical parameters including central tendency (e.g. means) or other basic estimates (e.g. regression coefficient)

AND variation (e.g. standard deviation) or associated estimates of uncertainty (e.g. confidence intervals)

$\varnothing$ For null hypothesis testing, the test statistic (e.g. $F, t, r$ ) with confidence intervals, effect sizes, degrees of freedom and $P$ value noted Give $P$ values as exact values whenever suitable.

\ $\square$ For Bayesian analysis, information on the choice of priors and Markov chain Monte Carlo settings

$\bigotimes \square$ For hierarchical and complex designs, identification of the appropriate level for tests and full reporting of outcomes

$\bigotimes$ Estimates of effect sizes (e.g. Cohen's $d$, Pearson's $r$ ), indicating how they were calculated

Our web collection on statistics for biologists contains articles on many of the points above.

\section{Software and code}

Policy information about availability of computer code

Data collection Data about vaccination behaviors and characteristics of participants in randomized controlled trials were collected from UCLA Health records. Data about online participants' responses to text messages and perceptions of COVID-19 and the vaccines were collected via Qualtrics.

Data analysis Data analysis was conducted in Stata 14 and R 4.1.0.

For manuscripts utilizing custom algorithms or software that are central to the research but not yet described in published literature, software must be made available to editors and reviewers. We strongly encourage code deposition in a community repository (e.g. GitHub). See the Nature Portfolio guidelines for submitting code \& software for further information.

\section{Data}

Policy information about availability of data

All manuscripts must include a data availability statement. This statement should provide the following information, where applicable:

- Accession codes, unique identifiers, or web links for publicly available datasets

- A description of any restrictions on data availability

- For clinical datasets or third party data, please ensure that the statement adheres to our policy

The two RCTs were pre-registered at clinicaltrials.gov (First RCT: https://clinicaltrials.gov/ct2/show/NCT04800965; Second RCT: https://clinicaltrials.gov/ct2/show/ NCT04801524). The three online experiments were pre-registered at aspredicted.org (Online Experiment 1: https://aspredicted.org/blind.php?x=u2ng5c, Online Experiment 2: https://aspredicted.org/blind.php?x=ae3ci5, and Online Experiment 3: https://aspredicted.org/blind.php? $\mathrm{x}=7 \mathrm{wf9er}$ and https://aspredicted.org/ blind.php?x=u82hy5). The data analyzed in this paper about randomized controlled trials was provided by UCLA Health and contains protected health information. To protect patient privacy, we cannot publicly post individual-level data. Qualified researchers with a valuable research question and relevant approvals including ethical approval can request access to the de-identified data about these trials from the corresponding author. A formal contract will be signed and an independent 


\section{Field-specific reporting}

Please select the one below that is the best fit for your research. If you are not sure, read the appropriate sections before making your selection.

Life sciences

Хehavioural \& social sciences Ecological, evolutionary \& environmental sciences

For a reference copy of the document with all sections, see nature.com/documents/nr-reporting-summary-flat.pdf

\section{Behavioural \& social sciences study design}

All studies must disclose on these points even when the disclosure is negative.

Study description

Research sample

Sampling strategy

Data collection

Timing
Study designs are outlined in the Methods section. In two randomized controlled trials (RCT), we varied whether patients received a text reminder as well as the type of reminder they got, and assessed whether they subsequently scheduled an appointment for the COVID-19 vaccine and eventually obtained the vaccine. In three online experiments, we presented participants with one of four text reminders used in our first RCT, and assessed their interest in getting the COVID-19 vaccine and their perceived persuasiveness of the reminder. These data are all quantitative experimental.

The research sample for the RCTs consist of UCLA Health patients who were eligible for the COVID-19 vaccine in January and February 2021, were 18 years old or older, and had not obtained the first dose of COVID-19 vaccine anywhere (as far as UCLA Health could tell) or made a first-dose vaccine appointment at UCLA Health at the time of enrollment in our RCTs. The analysis sample included in the first RCT has 93.354 patients who were enrolled by February 23, 2021 and fit our pre-registered exclusion criteria (43.3\% male, 53.5\% White (excluding Hispanic or Latino), average age=72.8, s.d.=10.3). The analysis sample for the second RCT includes 67,092 patients who were enrolled by February 23, 2021 and fit our pre-registered exclusion criteria (43.5\% male, 52.6\% White (excluding Hispanic or Latino), average age $=73.7$, s.d. $=10.0$ ). We chose UCLA patients as our study sample because UCLA Health is one of the largest healthcare systems in California (which allows us to assess a large patient population) and was supportive of evaluating the effectiveness of reminders in promoting COVID-19 appointment scheduling and uptake. Our RCT sample is not representative. See Extended Data Table 7 for a comparison of demographics and vaccination rates between our RCT sample, UCLA Health patients in general, Los Angeles County residents, and California residents.

The research sample for the online experiments consists of US participants from Amazon Mechanical Turk (MTurk) and Prolific Academic (Prolific) who (1) were 18 years old or older, (2) had not taken the COVID-19 vaccine or scheduled an appointment for the COVID-19 vaccine at the time of our studies, and (3) passed a Captcha and an attention check question at the beginning of a given study. Across the two online experiments conducted in February 2021, our final sample after data exclusion (described below) consists of 2,003 participants (51.0\% female, 71.8\% White (excluding Hispanic or Latino), 51.8\% Democrats, average age=37.9, s.d. =13.4). Our online experiment conducted in April 2021 consists of 1,178 participants ( $53.4 \%$ female, $71.6 \%$ White (excluding Hispanic or Latino), 40.8\% Democrats, average age=36.7, s.d.=12.0) after data exclusion (described below). These samples are not representative and are "convenience samples" from online survey platforms that social scientists commonly use.

For the RCTs, we enrolled all UCLA Health patients who met the eligibility criteria by the end of February 23, 2021 (a pre-registered date). The exact sample size could not be determined before RCTs started since we did not know in advance how many patients would fit the eligibility criteria by our pre-registered stopping date. Based on conversations with UCLA Health, we did expect to have at least 30,000 patients enrolled in the first RCT by the end of February 23, 2021, so we knew we had at least an $80 \%$ power to detect a 2-percentage-point difference between the Holdout arm and the Follow-Through Text Reminder arm, assuming that the Holdout arm would have a $50 \%$ baseline (two-sided proportion test, alpha $=0.05$ ). In the end, our analysis of the first RCT includes 93,354 patients, providing an $80 \%$ power to detect a 1.44-percentage-point difference between the Holdout arm and the Text Reminder arm, assuming that the Holdout arm would have a 50\% baseline. We were more uncertain about sample size for the second RCT (since we did not know how many people would still choose not to get vaccinated after already receiving a text reminder). Thus, we pre-registered analysis plans contingent on the number of patients enrolled in the second RCT by the end of February 23, 2021. In the end, our analysis of the second RCT includes 67,092 patients, providing an $80 \%$ power to detect a 1.54-percentage-point difference between the Holdout arm and the Follow-Through Text Reminder arm, assuming that the Holdout arm would have a 50\% baseline. In both RCTs, all patients who fit our pre-registered inclusion/exclusion criteria were enrolled, and there was no sampling from a larger eligible pool.

For the online experiments, we aimed to have at least 800 participants in each experiment, in order to have an $80 \%$ power to detect a main effect of video or ownership framing that is as large as Cohen's d of 0.2 (two-sided, alpha $=0.05$ ). The sampling procedure was convenience based: Participants opted into our studies; and once our target sample size was hit for a given study, the study was closed.

For the RCTs, data was collected from UCLA Health; no researchers were present for data collection, as enrollment and text message delivery were implemented by UCLA Health and an outside vendor who were blind to the hypotheses. For online experiments, data was collected using online survey software Qualtrics, which completed the randomization into separate experimental conditions; researchers were blind to experimental condition at the data collection stage.

Participants for the first RCT were enrolled from February 1, 2021 to February 23, 2021. Participants for the second RCT were enrolled from February 9, 2021 to February 23, 2021. Data on vaccination records and participant characteristics were extracted on May 25, 2021. For online experiments, data were collected from 2/21/2021 to 2/23/2021 for the first experiment, from 2/24/2021 to 2/25/2021 for the second experiment, and from 4/23/2021 to 4/29/2021 for the third experiment. More details about each sample can be found in Methods and the Supplementary information. 
For the first RCT, among 132,337 patients enrolled from February 1, 2021 to February 23, 2021, we excluded 33,533 patients who obtained the first dose somewhere before the first reminder date, 5,392 patients who made the first-dose appointment at UCLA Health before 3pm PST, and 58 patients who were below 18 years old. For the second RCT, among 102,675 patients enrolled from February 9, 2021 to February 23, 2021, we excluded 35,127 patients who obtained the first dose somewhere before the second reminder date, 408 who made the first-dose appointment at UCLA Health before 3pm PST, and 48 who were below 18 years old. These exclusions were all pre-registered and implemented using the latest records extracted on May 25, 2021.

The first online experiment excluded participants who reported having technical problems with the video, or did not complete our pre-registered dependent variables. The second experiment had the same exclusion criteria but additionally excluded Prolific participants who had taken a similar study on Amazon Mechanical Turk. The third experiment had the same exclusion criteria as the second experiment. All of these criteria were pre-registered, except that for the first experiment, we did not pre-register to exclude participants who reported having technical problems with the video. However, since we combined the first and second experiments (both conducted in February concurrently to our RCTs) in our main analysis, we adopted this exclusion criterion (which was preregistered for the second experiment) consistently for both experiments.

Non-participation

Randomization
For the RCTs, all participants who fit our eligibility criteria were automatically enrolled, and nobody dropped out. For online experiments, no participants requested to withdraw their responses.

Participants in the first RCT were randomly assigned at a 4:1 ratio to either the Follow-Through Reminder arm or the Holdout arm. Patients in the Follow-Through Reminder arm were randomly assigned with an equal probability to one of four subarms: basic reminder, basic reminder with video, ownership reminder, and ownership reminder with video. Participants in the second RCT were randomly assigned at a 6:1 ratio to either the Follow-Through Reminder arm or the Holdout arm. Patients in the Follow-Through Reminder arm were randomly assigned with an equal probability to one of six subarms: basic self, basic prosocial, early access self, early access prosocial, fresh start self, and fresh start prosocial.

Participants in the online experiments were randomly assigned with an equal probability to read one of the four text messages from our first RCT. In the third experiment, participants were also randomly assigned with an equal probability to answer one of two types of vaccination intention measures.

\section{Reporting for specific materials, systems and methods}

We require information from authors about some types of materials, experimental systems and methods used in many studies. Here, indicate whether each material, system or method listed is relevant to your study. If you are not sure if a list item applies to your research, read the appropriate section before selecting a response.

\begin{tabular}{l|l} 
Materials \& experimental systems \\
\hline $\mathrm{n} / \mathrm{a}$ & Involved in the study \\
\hline & $\square$ Antibodies \\
\hline & $\square$ Eukaryotic cell lines \\
$\square$ & $\square$ Palaeontology and archaeology \\
$\square$ & $\square$ Animals and other organisms \\
$\square$ & $\square$ Human research participants \\
$\square$ & $\square$ Clinical data
\end{tabular}

Methods

\section{Human research participants}

Policy information about studies involving human research participants

Population characteristics

Recruitment
See above, the Methods, and Supplementary Information for more information about participant characteristics

Our RCTs are part of the vaccination outreach effort at UCLA Health. Starting from January 19, 2021, UCLA Health invited patients who were eligible for the COVID-19 vaccine at the time to get vaccinated. UCLA Health sent out invitations to patients in batches. On the first weekday following the initial invitation, eligible patients were automatically enrolled in the first RCT. On the first weekday after the eighth day following the initial invitation, eligible patients were automatically enrolled in the second RCT. Eligibility criteria are described above in Behavioral \& Social Sciences study design. Note that since the infrastructure needed to run the RCTs was not ready until February 2021, patients who received the initial invitation during January 19-29, 2021 were enrolled in the first RCT on February 1, 2021 and in the second RCT on February 9, 2021. All other batches of patients were enrolled in the first and/or second RCT based on the aforementioned timeline. Regarding self-selection bias, all patients who fit our enrollment inclusion/exclusion criteria were automatically enrolled and randomized to condition; since patients could not withdraw from the RCTs, no patient was lost to follow up, and treatment could not affect the inclusion/exclusion criteria for our analysis sample, randomization and causal inference are maintained for our analysis sample. See Extended Data Tables 1 and 3 for balance check across conditions.

For the first online experiment, participants were recruited on MTurk and Prolific from 2/21/2021 to 2/23/2021 in exchange for $\$ 0.90$ and $\$ 1.10$, respectively. For the second online experiment, participants were recruited on Prolific from 2/24/2021 to $2 / 25 / 2021$ in exchange for $\$ 1.10$. For the third experiment, participants were recruited on MTurk from 4/23/2021 to $4 / 29 / 2021$ in exchange of $\$ 0.90$ or $\$ 1.00$ (We boosted the pay to $\$ 1.00$ on the third day of our data collection to attract 
Ethics oversight

more respondents) and Prolific from 4/28/2021 to 4/29/2021 in exchange of \$1.10. Regarding self-selection bias, due to informed consent procedures and the use of brief advertisements, people may have chosen to participate based on their knowledge of or interest in our survey topic. This is true for any survey study that involves participant consent. Because participants were randomly assigned to condition, it is unlikely self-selection would result in the effects observed in our online experiment.

This research was deemed to comply with all relevant ethical regulations. The Institutional Review Board at the University of California Los Angeles approved the protocols of our randomized controlled trials (reference number 21-000268) and determined that a waiver of informed consent was appropriate. All online experiments and the vaccination intention survey were conducted under approval of the Institutional Review Board at Carnegie Mellon University (reference number IRBSTUDY2015_00000482), and informed consent was obtained from all online study participants as part of the enrollment process.

Note that full information on the approval of the study protocol must also be provided in the manuscript.

\section{Clinical data}

Policy information about clinical studies

All manuscripts should comply with the ICMJE guidelines for publication of clinical research and a completed CONSORT checklist must be included with all submissions.

Clinical trial registration The First RCT: https://clinicaltrials.gov/ct2/show/NCT04800965; The Second RCT: https://clinicaltrials.gov/ct2/show/NCT04801524

Study protocol

Data collection

Outcomes
Detail about the full trial protocol (the exact content of text messages, access to the video, and access to the survey where the video was embedded) is available in Methods and Supplementary Information.

Please see above in the Behavioral \& Social Sciences Study Design

Our pre-registered primary outcome measure indicates whether patients scheduled a first-dose appointment at UCLA Health within six days of the first (second) reminder date. We pre-registered this time window because UCLA Health targeted additional outreach efforts to patients who had not scheduled their vaccination appointment six days after the second reminder date and we wanted to use a consistent time window for the two RCTs. Our secondary outcome measure in this paper is whether patients obtained the vaccine at UCLA Health within four weeks of the first (second) reminder date. We chose this window because UCLA Health generally only allowed patients to schedule an appointment for less than four weeks ahead. Consistent with this practice, $96.25 \%$ of the firstdose appointments made by patients in the analysis sample of the first RCT occurred within four weeks from the day they were scheduled. In the pre-registrations, we listed additional secondary outcome variables; we explained in Supplementary Information why we did not focus on these in this paper. 